\title{
Six host-range restricted poxviruses from three genera induce distinct gene expression profiles in an in vivo mouse model
}

Kristy Offerman ${ }^{1,3}$, Armin Deffur ${ }^{2,4}$, Olivia Carulei ${ }^{1,3}$, Robert Wilkinson ${ }^{2,3,5,6}$, Nicola Douglass ${ }^{1,3}$ and Anna-Lise Williamson ${ }^{1,3,7^{*}}$

\begin{abstract}
Background: Host-range restricted poxviruses make promising vaccine vectors due to their safety profile and immunogenicity. An understanding of the host innate immune responses produced by different poxvirus vectors would aid in the assessment, selection and rational design of improved vaccines for human and veterinary applications. Novel avipoxviruses are being assessed to determine if they are different from other poxvirus vectors. Analysis of the transcriptome induced in a mouse model would aid in determining if there were significant differences between different poxvirus vectors which may reflect different adjuvant potential as well as establish if they should be further evaluated as vaccine vectors.

Results: We compared host transcript abundance in the spleens of BALB/C mice twenty four hours after intravenous infection ( $10^{5} \mathrm{pfu} / \mathrm{mouse}$ ) with six host-restricted poxvirus species from three genera, namely Lumpy Skin Disease virus (LSDV), Canarypox virus (CNPV), Fowlpox virus (FWPV), modified vaccinia Ankara (MVA) and two novel South African avipoxviruses, Feral Pigeonpox virus (FeP2) and Penguinpox virus (PEPV). These six viruses produced qualitatively and quantitatively distinct host responses with LSDV, followed by MVA, inducing the greatest interferon (IFN) response. FeP2 and PEPV caused very little change to host transcript abundance compared to the other 4 viruses tested. CNPV and FWPV induced the up regulation of two immunoglobulin genes (lghg and lghg3 (lgG3)) with CNPV inducing a third, Ighm (IgM). HIV-1-specific lgG3 antibodies have been correlated with decreased risk of HIV-1 infection in the RV144 trial, which included a CNPV-based vector (Yates et al. (Sci Transl Med, 6(228) p228, 2014). Up regulation of lgG3 by CNPV and FWPV but not the other poxviruses tested in vivo, implies that these two avipoxvirus-vector backbones may be involved in stimulation of the clinically important IgG3 antibody subclass. Differential transcript abundance associated with the different poxviruses is further discussed with particular emphasis on responses related to immune responses.

Conclusion: Six, genetically diverse host-restricted poxviruses produce different responses in a mouse model early after infection. These differences may affect the immune response induced to vaccine antigen in vectors based on these viruses. The two novel avipoxviruses were clearly distinguishable from the other viruses.
\end{abstract}

Keywords: Poxvirus, Vaccine, Micro-array, LSDV, MVA, Avipoxviruses

\footnotetext{
* Correspondence: anna-lise.williamson@uct.ac.za

'Division of Medical Virology, Department of Clinical Laboratory Sciences,

University of Cape Town, Cape Town, South Africa

${ }^{3}$ Institute of Infectious Disease and Molecular Medicine, University of Cape

Town, Cape Town, South Africa

Full list of author information is available at the end of the article
} 


\section{Background}

Although a number of different poxvirus-based vaccine vectors are available [1-7], there is still a need for additional vaccine vectors as well as improvement of the vectors. The unique response elicited by the host to different vectors means that vectors can be selected or engineered according to a desired host response. Host-range restricted poxviruses have been shown to successfully activate the host immune system $[8,9]$ and evidence exists that each virus does this in a different way, with an accompanying different pattern of transcript abundance [10-15]. The poxviruses ALVAC (based on canarypox virus), modified vaccinia Ankara (MVA) and NYVAC (both based on vaccinia virus (VACV) and have specific deletions) produce distinct innate immune profiles, characterised by different induction of pro-inflammatory and antiviral cytokines and chemokines in both rhesus monkeys and human PBMC [16].

It has been shown that in non-permissive cells, Fowlpox virus (FWPV) proceeds further into the poxvirus life cycle than Canarypox virus (CNPV) [17]. Heterologous HIV gag/pol and env genes are more efficiently expressed by FWPV than CNPV in vitro due to longer transgene expression [18]. However, the only successful HIV-1 vaccine clinical trial to date $(31.2 \%$ protection from HIV-1 infection) has been the Thai RV144 trial involving priming with ALVAC expressing HIV-1 gp120/Gag-Pro and boosting with a recombinant glycoprotein 120 subunit, AIDSVAX [7]. Head to head comparisons of poxvirusvectored vaccines would help to establish the differences between the different vaccine vectors and the vaccineinduced response to achieve protection against pathogens and cancers.

Innate immunity is critical for directing the adaptive immune response to antigen and influences the magnitude and quality of the long-lived, protective immune responses to pathogens or vaccines [19]. Application of the systems biology approach to vaccine development ("systems vaccinology") and establishment of innate immune signatures has proven useful in predicting the immunogenicity of the highly effective yellow fever vaccine (YF-17D) [20], seasonal influenza vaccines [21] and the immunogenic but inefficacious Merck Adenovirus type 5 (Ad5) based HIV vaccine [22]. A better understanding of the mechanisms underlying the optimal innate immune responses would aid rational vaccine development.

Type 1 interferons (IFN $\alpha / \beta)$ are expressed rapidly in response to viral infection, and, in turn activate many interferon stimulated genes (ISGs) which exert various antiviral effector functions. A fine balance of IFN is required for successful vaccination using a live virus vector. The vector should induce enough type I IFNs to activate the immune system, yet not enough to inhibit viral DNA replication and gene expression before antigen presentation can occur [23]. This is corroborated by
Johnson et al. (2012), who compared recombinant (r) Ad types 5,28 and 35 . Specific IFN- $\alpha$ induction by rAd28 and rAd35 significantly lowered the immunogenicity of these vectors compared to rAd5 which did not induce IFN- $\alpha$ expression [24]. The effect of type 1 IFN responses on different vaccines requires delineation of innate immune signatures and how they determine subsequent adaptive responses.

Microarray analyses performed in vitro have been used to investigate the effects of VACV ([12], MVA [11] and NYVAC [13] infection on HeLa cell gene expression. Gene expression profiles in human monocyte derived dendritic cells (MDDCs) have also been generated with MVA, NYVAC [14] and ALVAC [15]. Furthermore, a comparison of the closely related VACV-derived vectors NYVAC and MVA revealed significant differences in antigen production and host gene dysregulation in cell culture [25]. Consequently we hypothesized that genetically diverse poxvirus strains would induce significant differences in host gene expression. The interaction of poxviruses with the host is not just dependent on the actual cell infected by the virus but also on the factors secreted by those infected cells and their effects on the surrounding cells. Although in vitro expression studies have provided useful information, gene expression profiles performed in cell culture may not accurately reflect the changes in the system that occurs as a result of infection in vivo. A recent study in Rhesus Macaques showed that ALVAC induced distinct cytokine and chemokine levels compared to the vaccinia virus-based vectors MVA and NYVAC and that multiple subsets of peripheral blood mononuclear cells (PBMC) are likely to contribute to the overall response to different poxviruses [16].

In this study we compared the effects of the capripoxvirus, lumpy skin disease virus (LSDV), the orthopoxvirus, MVA, and the avipoxviruses, CNPV, FWPV, a novel pigeonpox virus (FeP2) $[26,27]$ and a novel penguinpox virus (PEPV) [27-29] on host gene expression profiles in the spleens of BALB/c mice. None of these viruses complete their replication cycle in mice.

\section{Results}

Comparison of the host responses to different poxviruses We compared the differential host gene expression induced by six host-restricted poxviruses, MVA, LSDV, FWPV, CNPV, FeP2 and PEPV, in the spleens of BALB/c mice $24 \mathrm{~h}$ post infection. Transcripts with an adjusted $p$ value $<0.05$ were described as up-regulated if they had a $\log _{2}$ fold change (FC) of $\geq 1$, or down-regulated if they had a $\log _{2} \mathrm{FC}$ of $\leq-1$. A summary of the number of up and down-regulated genes is given in Table 1. Full gene lists are given in supplementary data (Additional file 1). Quantitative RT-PCR showed all three housekeeping genes (GAPDH, HPRT and CD51) to be expressed at 
similar levels to those of the PBS control for all samples. Both IRF7 and Zbp1 were upregulated by all poxviruses tested. IGFbp3 was shown to be downregulated by all viruses. Overall the quantitative RT-PCR was more sensitive than the microarray, but the trend observed in upand down-regulation of host gene expression was similar for qRT-PCR and microarray analysis.

Unsupervised hierarchical clustering based on the genes with $p$-value $<0.05$ and $\log _{2} \mathrm{FC}$ above or below cutoff $(>1$, $<-1)$ showed that each virus induced a unique overall response (Fig. 1). Venn diagrams highlight the number of differences and similarities in the up- and down-regulated genes between the viruses (Fig. 2). Fig. 2 a and b show the differences in transcripts up (A) and (B) down-regulated respectively between FWPV, CNPV, MVA and LSDV. The Venn diagrams comparing FWPV, CNPV, MVA and LSDV indicate that the majority of up-regulated genes are shared amongst these 4 viruses (Fig. 2). The downregulated genes however, appear largely unique, especially for LSDV and MVA (Fig. 2). FWPV and CNPV down regulate a smaller number of genes in comparison to LSDV and MVA. Comparison of avipoxvirus-induced upand down-regulated genes shows that FeP2 and PEPV induce significantly less change in host transcript abundance than FWPV and CNPV (Fig. 2). FeP2 induced the lowest response (Fig. 2, Table 1). For all six viruses, more genes were up-regulated than down-regulated (Table 1).

\section{Histone transcripts}

Previous studies have found that increased detection of histone genes by poxvirus infection is described as an experimental artefact due to the de novo polyadenylation of transcripts by the viral poly-A polymerase [30,31]. Several histone transcripts (39 in total) were down-regulated in response to virus infection and, because any interpretation of these transcripts would be speculative, these have been excluded from further analysis.

\section{Immunity and host defence response-related genes Up-regulated immune response genes}

Selected up-regulated genes involved in the immune response are listed in Table 2. (Full list of up-regulated genes is given in Additional file 1). Seventeen of these genes are uniquely up-regulated by LSDV. RIG-I (Ddx58) senses viral nucleic acid [32], Cebpb is important for macrophage function [33] and control of inflammatory responses [34], Tap1 and Tap2 genes are involved in antigen presentation to MHC class 1 molecules [35], Ifitm3 and Ifi203 are interferon responsive genes (ISG), c-Myc and Mif are transcription factors and Adar is an RNA editing enzyme. There are eight genes induced by CNPV, FWPV and MVA, which were not up-regulated in LSDV-infected mice. Two of these include the cytidine deaminase, Apobec1, which can edit viral nucleic acid and can thereby limit viral replication [36], and Caspase 1 (Casp1), which is associated with pyroptosis (Table 2).

Twenty six genes involved in the host immune/defence response were up-regulated only in avipoxvirus-infected mouse spleens (Table 2). The only avipoxvirus-specific gene that was up-regulated by all four avipoxviruses was the macrophage receptor with collagenous structure (Marco) gene which has been shown to suppress early inflammatory responses to virus infection [37]. There were, however, 9 additional genes which were up-regulated by both CNPV and FWPV that were not induced by the other viruses. These included the chemokine Ccl6 which promotes immune cell activation and recruitment [38] and the immunoglobulin heavy chain genes, Ighg (IgG) and Ighg3 (IgG3) (Table 2). Amongst these 26 avipoxvirusspecific genes, 14 were exclusively up-regulated by FWPV. The Nod-like receptor, NLR, Nod1, which has been shown to be augmented in response to virus-induced production of type I IFNs [39] was exclusively up-regulated by FWPV. Four genes were uniquely up-regulated in CNPV-infected mice including the immunoglobulin heavy chain gene, Ighm (IgM), lymphocyte antigen 96 (Ly96), proteasome maturation protein (Pomp) and Cathepsin L (Ctsl) (Table 2). $\mathrm{PePV}$ and FeP2 induced very little immune activation according to this microarray analysis.

Four genes were up-regulated by LSDV and MVA that were not induced by the avipoxviruses in mice (Table 2), namely the Moloney Leukemia Virus 10 (Mov10) gene, hematopoietic $\mathrm{SH} 2$ domain containing protein (Hsh2d), poly (ADP-ribose) polymerase family, member 11 (Parp11) and schlafen 8 (Slfn8). No genes were uniquely upregulated in response to MVA infection (Table 2).

\section{Down-regulated immune related genes}

Selected down-regulated genes involved in the immune response are listed in Table 3. Full lists of down-regulated genes in response to each virus are given in Additional file 1. MVA and LSDV induced the down regulation of several genes that were not affected in avipoxvirus-infected spleens. These included three forms of the chemokine CCL21 (Ccl21a, Ccl21b, Ccl21c) which are potent chemoattractants for lymphocytes and dendritic cells [40] (Table 3). Furthermore, MVA and LSDV down regulate the high affinity IgM and IgA $F_{C}$ receptor Fcamr. Fcamr is the receptor for the $F_{C}$ fragment of immunoglobulins IgA and IgM [41]. Interestingly, MVA, LSDV, FWPV and CNPV all down regulate the gene encoding the murine homolog for DC-specific ICAM-3-grabbing nonintegrin (DC SIGN) (Cd209a), and MVA and LSDV down regulate an additional DC SIGN homolog, CD209b (SIGNR1) (Table 3). LSDV uniquely down regulates CD59a, which is the primary regulator of complement membrane attack in mouse [42] and CD7 which is expressed on T- and NK cells, and on cells in the early stages of T, B, and myeloid 
Table 1 Summary of the number of significantly up- and down-regulated transcripts with adjusted $p$-value $<0.05$

\begin{tabular}{lll}
\hline & Up-regulated $\log _{2} F C>1$ & Down-regulated $\log _{2} F C<-1$ \\
\hline MVA & $299(42 \mathrm{NA})$ & $177(86 \mathrm{NA})$ \\
LSDV & $463(111 \mathrm{NA})$ & $85(11 \mathrm{NA})$ \\
FWPV & $433(101 \mathrm{NA})$ & $62(28 \mathrm{NA})$ \\
CNPV & $280(31 \mathrm{NA})$ & $47(11 \mathrm{NA})$ \\
FeP2 & $20(1 \mathrm{NA})$ & $3(0 \mathrm{NA})$ \\
PEPV & $68(6 \mathrm{NA})$ & $19(2 \mathrm{NA})$ \\
\hline
\end{tabular}

Genes are described as upregulated if they had a fold change of $\geq 2$, or downregulated if they had a $\log _{2}$ Fold change of $\leq-1$. These included genes that are not annotated and therefore do not have an Entrez ID. The number of genes without annotation are indicated in brackets

cell differentiation [43]. LSDV also uniquely down regulates the immunoglobulin kappa chain complex (IgK) amongst other immune related genes (Table 3). TLR11 is downregulated by MVA alone (Table 3 ).

\section{Type I interferon response}

While many of the immunity related genes listed in Table 2 are regulated in some way by Type I Interferons, in order to characterise and compare the differences in the Type I IFN-regulated responses between MVA, LSDV, FWPV, CNPV, FeP2 and PEPV at $24 \mathrm{~h}$, we analyzed a selection of genes known to be involved in the IFN response [44-46] (Fig. 3). This figure clearly shows that LSDV induces the greatest IFN response compared to the other viruses (Fig. 3).

\section{Caspases}

MVA, FWPV and CNPV all up-regulated the protease caspase 1 (casp1) whereas LSDV did not (Fig. 4). MVA, FWPV, CNPV and LSDV significantly up-regulated caspase 4 (casp 4) (historically called caspase 11 in the mouse) (Table 2). The SA avipoxviruses, FeP2 and PEPV did not affect gene regulation of any caspase genes.

\section{$B$ - and T-cell specific responses}

The up- and down-regulated genes involved in B cell and $\mathrm{T}$ cell responses induced by the viruses in this study were compared (Fig. 4). Fig. 4a highlights the regulated genes that are involved in the $\mathrm{T}$ cell response. FeP2 and PEPV regulated only one gene each involved in this response, Ctla2a and major histocompatibility (MHC) class I gene, H2-M2, respectively. The other four viruses differentially regulated several MHC class I genes amongst others (Fig. 4a). As highlighted in Table 2, the avipoxviruses, CNPV and FWPV, exclusively up regulate immunoglobulin heavy chain genes, (Ighg (IgG) and Ighg3 (IgG3)) with CNPV inducing a third, Ighm (IgM) (Fig. 4b). LSDV down regulates the immunoglobulin kappa (IgK) chain complex (Fig. 4b). In addition to these, the poxviruses differentially regulate genes for $F_{C}$ receptors and complement (Fig. 4b).

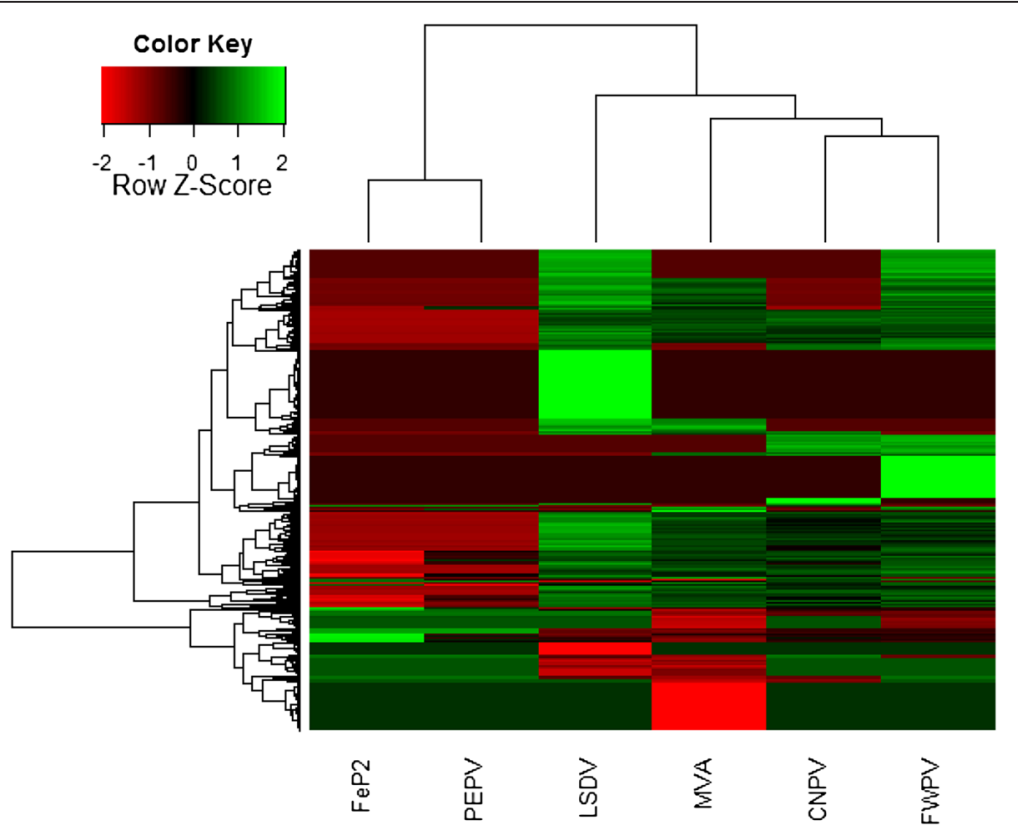

Fig. 1 Heatmap comparing the differential expression induced in mouse spleens in response to pigeonpox (FeP2), penguinpox (PEPV), lumpy skin disease virus (LSDV), modified vaccinia Ankara (MVA), canarypox virus (CNPV) and fowlpox virus (FWPV). Only genes (with $p$-value $<0.05$ ) with $\log _{2}$ fold change induction above or below the cutoff $( \pm 1)$ as compared to the mock infected control are shown. Unsupervised hierarchical clustering of the samples is represented by dendograms. Clustering analysis and heatmap was performed in the R package, gplots (Warnes, 2009) 


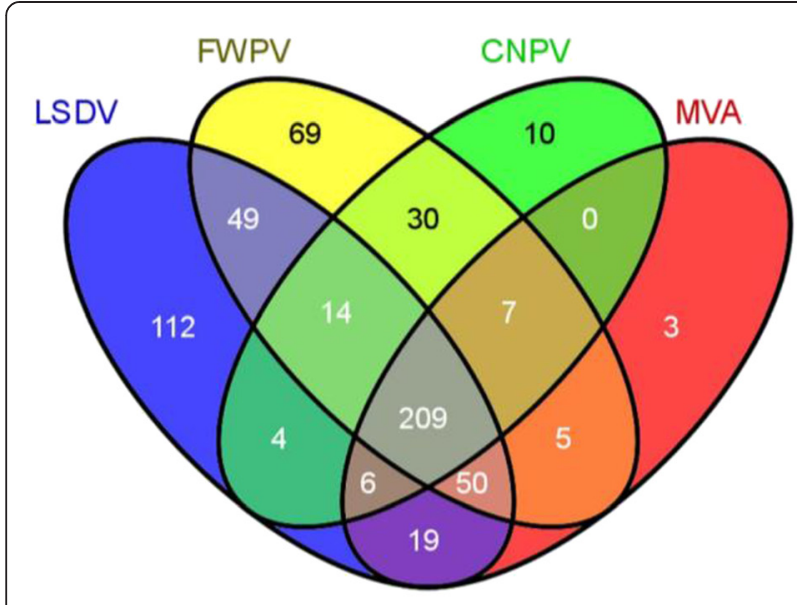

A. Upregulated genes

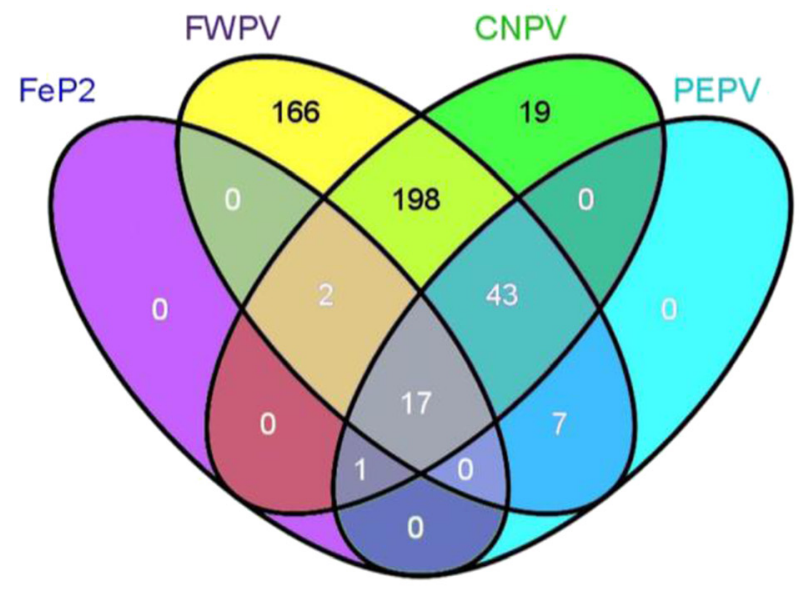

C. Avipoxvirus-induced upregulated genes

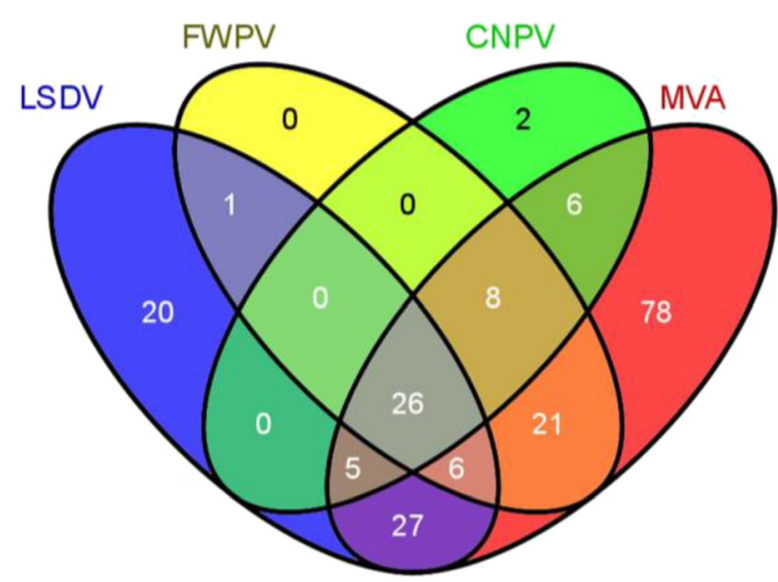

B. Downregulated genes

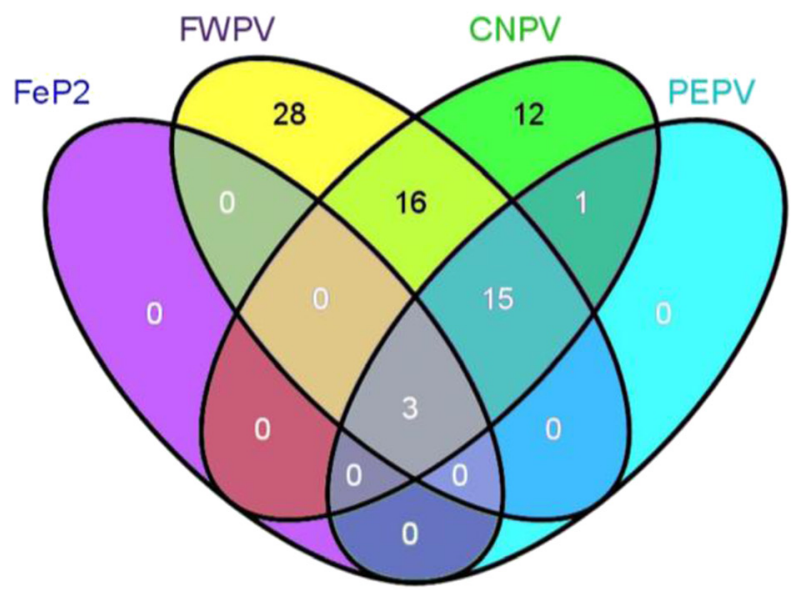

D. Avipoxvirus-induced downregulated genes

Fig. 2 Venn diagrams showing the overlap between the differentially up-regulated (a) and down-regulated (b) transcripts induced by canarypox virus (CNPV), fowlpox virus (FWPV), modified vaccinia Ankara (MVA) and lumpy skin disease virus (LSDV) and the up-regulated (c) and down-regulated (d) transcripts induced by the four avipoxviruses. For each diagram, the circles represent the number of differently expressed transcripts regulated by each virus ( $p$ value $\leq 0.05, \log _{2}$ fold change of $\geq \pm 1$ ). The numbers in the intersections of each circle represents the number of transcripts common to the respective virus/es

\section{Comparison of early poxvirus-induced immune responses to} innate molecular signatures of published candidate vaccine vectors

We compared the gene expression induced by each of the 6 poxviruses at $24 \mathrm{~h}$ to selected correlates of protection and molecular signatures from previously published studies (Table 4). The induction of multiple PRRs has been shown to activate different immune pathways and thereby induce a more polyvalent immune response [47, 48]. We identified differential expression of several genes involved in pathogen recognition (Table 4). Several genes are common to the innate and adaptive immune responses induced by the poxviruses analysed here and other viral vectors analysed elsewhere (Table 4).

\section{Discussion}

Novel avipoxviruses have been isolated in South Africa with the goal of identifying novel vaccine vectors [26, 27]. It is desirable to be able to select potential avipoxvirus vaccine vectors without going through the process of making recombinant viruses and testing immunogenicity in animal models. In this study, we compared the gene expression profiles in mouse spleens $24 \mathrm{~h}$ after infection with six poxviruses from 3 different genera. All the viruses were grown in eggs and the same purification methodology used. There have been no comparisons of host responses to these six different poxviruses. One of the aims of this study was to determine if one could select novel poxvirus vaccine vectors based on the transcriptome analysis. It was hypothesized that if the 
Table 2 Selection of up-regulated genes in mouse spleens in response to MVA, LSDV, CNPV, FWPV, PEPV and FeP2. Differences in $\log _{2}$ Fold Changes (between each virus and the control) are depicted.

\begin{tabular}{|c|c|c|c|c|c|c|c|c|}
\hline Symbol & Name & Entrez & MVA & LSDV & CNPV & FWPV & PEPV & $\mathrm{FeP} 2$ \\
\hline \multicolumn{9}{|c|}{ Genes induced by LSDV alone } \\
\hline Oasib & 2'-5' oligoadenylate synthetase $1 B$ & 23961 & - & 1.6 & - & - & - & - \\
\hline Adar & adenosine deaminase, RNA-specific & 56417 & - & 1 & - & - & - & - \\
\hline Cebpb & CCAAT/enhancer binding protein (C/EBP), beta & 12608 & - & 1 & - & - & - & - \\
\hline$D d \times 58(R / G-1)$ & DEAD (Asp-Glu-Ala-Asp) box polypeptide 58 & 230073 & - & 1.3 & - & - & - & - \\
\hline Grn & granulin & 14824 & - & 1.1 & - & - & - & - \\
\hline Gvin1 & GTPase, very large interferon inducible 1 & 74558 & - & 1.5 & - & - & - & - \\
\hline Gm17757 & GTPase, very large interferon inducible 1 pseudogene & 100417829 & - & 1.5 & - & - & - & - \\
\hline H2-T24 & histocompatibility 2, T region locus 24 & 15042 & - & 1.2 & - & - & - & - \\
\hline Ifi203 & interferon activated gene 203 & 15950 & - & 1.1 & - & - & - & - \\
\hline Ifitm3 & interferon induced transmembrane protein 3 & 66141 & - & 1.4 & - & - & - & - \\
\hline$|f i 27| 2 a$ & interferon, alpha-inducible protein 27 like $2 \mathrm{~A}$ & 76933 & - & 1.9 & - & - & - & - \\
\hline Ly6i & lymphocyte antigen 6 complex, locus I & 57248 & - & 1 & - & - & - & - \\
\hline Mif & macrophage migration inhibitory factor & 17319 & - & 1.1 & - & - & - & - \\
\hline Myc & myelocytomatosis oncogene & 17869 & - & 1.1 & - & - & - & - \\
\hline Nirc5 & NLR family, CARD domain containing 5 & 434341 & - & 1.8 & - & - & - & - \\
\hline Slfn2 & schlafen 2 & 20556 & - & 1.1 & - & - & - & - \\
\hline Stat1 & signal transducer and activator of transcription 1 & 20846 & - & 1.4 & - & - & - & - \\
\hline Tap 1 & transporter 1, ATP-binding cassette, sub-family B (MDR/TAP) & 21354 & - & 1.1 & - & - & - & - \\
\hline Tap2 & transporter 2, ATP-binding cassette, sub-family B (MDR/TAP) & 21355 & - & 1 & - & - & - & - \\
\hline Trim25 & tripartite motif-containing 25 & 217069 & - & 1 & - & - & - & - \\
\hline Trim34b & tripartite motif-containing $34 B$ & 434218 & - & 1.1 & - & - & - & - \\
\hline \multicolumn{9}{|c|}{ Genes induced by CNPV, FWPV and MVA only, and not LSDV. } \\
\hline Casp1 & caspase 1 & $\underline{12362}$ & $\underline{1.1}$ & - & 1.2 & 1.1 & 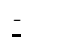 & - \\
\hline Clec $4 a 2$ & C-type lectin domain family 4 , member a2 & 26888 & 1.2 & z & 1.5 & 1.1 & - & - \\
\hline$\underline{\text { Ifi205 }}$ & interferon activated gene 205 & $\underline{226695}$ & 1.4 & $=$ & 1.6 & $\underline{1.8}$ & - & $=$ \\
\hline$\underline{\operatorname{Prd} x 1}$ & peroxiredoxin 1 & $\underline{18477}$ & 1 & $=$ & 1 & 1.2 & $=$ & $=$ \\
\hline$\underline{\text { Pnpt1 }}$ & polyribonucleotide nucleotidyltransferase 1 & $\underline{71701}$ & 1.1 & $=$ & $\underline{1.1}$ & 1.4 & $=$ & $=$ \\
\hline$\underline{\text { Scimp }}$ & $\underline{\text { SLP adaptor and CSK interacting membrane protein }}$ & 327957 & 1.2 & $=$ & 1.2 & 1.5 & $=$ & $=$ \\
\hline \multicolumn{9}{|c|}{ Genes induced by avipoxviruses only (CNPV, FWPV, FEP2 and/ or PEPV) } \\
\hline Anxa1 & annexin A1 & 16952 & - & - & 1.6 & 1.8 & - & - \\
\hline Apobec1 & apolipoprotein B mRNA editing enzyme, catalytic polypeptide 1 & 11810 & - & - & 1.2 & 1.2 & - & - \\
\hline Ccl6 & chemokine (C-C motif) ligand 6 & 20305 & - & - & 1.3 & 1.3 & - & - \\
\hline Ear2 & eosinophil-associated, ribonuclease A family, member 2 & 13587 & - & - & 1.3 & 1.5 & - & - \\
\hline Hsbp1 & heat shock factor binding protein 1 & 68196 & - & - & 1.2 & 1.2 & - & - \\
\hline lghg & Immunoglobulin heavy chain (gamma polypeptide) & 380794 & - & - & 1.5 & 1.6 & - & - \\
\hline lghg3 & Immunoglobulin heavy constant gamma 3 & 380795 & - & - & 1.3 & 1.2 & - & - \\
\hline Lilrb3 & $\begin{array}{l}\text { leukocyte immunoglobulin-like receptor, subfamily B } \\
\text { (with TM and ITIM domains), member } 3\end{array}$ & 18733 & - & - & 1.1 & 1 & - & - \\
\hline Marco & macrophage receptor with collagenous structure & 17167 & - & - & 1 & - & 1.5 & 1.5 \\
\hline Pf4 & platelet factor 4 & 56744 & - & - & 1.2 & 1.1 & - & - \\
\hline Pram1 & PML-RAR alpha-regulated adaptor molecule 1 & 378460 & - & - & 1.1 & 1.1 & - & - \\
\hline Psma1 & proteasome (prosome, macropain) subunit, alpha type 1 & 26440 & - & - & 1.1 & 1.3 & - & - \\
\hline
\end{tabular}

Genes induced by FWPV only. 
Table 2 Selection of up-regulated genes in mouse spleens in response to MVA, LSDV, CNPV, FWPV, PEPV and FeP2. Differences in $\log _{2}$ Fold Changes (between each virus and the control) are depicted. (Continued)

\begin{tabular}{|c|c|c|c|c|c|c|c|c|}
\hline Aif1 & allograft inflammatory factor 1 & 11629 & - & - & - & 1.1 & - & - \\
\hline Anxa2 & annexin $A 2$ & 12306 & - & - & - & 1.1 & - & - \\
\hline$D d x 18$ & DEAD (Asp-Glu-Ala-Asp) box polypeptide 18 & 66942 & - & - & - & 1.1 & - & - \\
\hline Den & decorin & 13179 & - & - & - & 1.5 & - & - \\
\hline Fgl2 & fibrinogen-like protein 2 & 14190 & - & - & - & 1.2 & - & - \\
\hline Gsdmd & gasdermin $D$ & 69146 & - & - & - & 1.2 & - & - \\
\hline Myd88 & myeloid differentiation primary response gene 88 & 17874 & - & - & - & 1.2 & - & - \\
\hline Nos2 & nitric oxide synthase 2 , inducible & 18126 & - & - & - & 1.1 & - & - \\
\hline Nod1 & nucleotide-binding oligomerization domain containing 1 & 107607 & - & - & - & 1 & - & - \\
\hline Pdcd5 & programmed cell death 5 & 56330 & - & - & - & 1.2 & - & - \\
\hline Psmc6 & proteasome (prosome, macropain) $26 \mathrm{~S}$ subunit, ATPase, 6 & 67089 & - & - & - & 1 & - & - \\
\hline Prmt1 & protein arginine $\mathrm{N}$-methyltransferase 1 & 15469 & - & - & - & 1 & - & - \\
\hline Serpinb6b & serine (or cysteine) peptidase inhibitor, clade B, member $6 b$ & 20708 & - & - & - & 1.2 & - & - \\
\hline \multicolumn{9}{|c|}{ Genes induced by CNPV only. } \\
\hline$\underline{\text { Ctsl }}$ & cathepsin L & $\underline{13039}$ & $=$ & 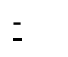 & $\underline{1.1}$ & 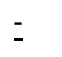 & 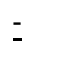 & $=$ \\
\hline$\underline{\text { Ighm }}$ & immunoglobulin heavy constant mu & $\underline{16019}$ & $=$ & - & $\underline{1.1}$ & $=$ & $=$ & $=$ \\
\hline$\underline{\text { Ly96 }}$ & lymphocyte antigen 96 & $\underline{17087}$ & $=$ & $=$ & $\underline{1.2}$ & $=$ & $=$ & $=$ \\
\hline Pomp & $\underline{\text { proteasome maturation protein }}$ & $\underline{66537}$ & $=$ & $=$ & 1 & $=$ & $=$ & $=$ \\
\hline \multicolumn{9}{|c|}{ Genes induced by LSDV AND MVA, but not by the AVIPOXVIRUSES } \\
\hline$\underline{\mathrm{Hsh} 2 \mathrm{~d}}$ & hematopoietic SH2 domain containing & $\underline{209488}$ & 1.1 & 1.4 & - & - & - & - \\
\hline Mov10 & $\underline{\text { Moloney leukemia virus } 10}$ & $\underline{17454}$ & $\underline{1.1}$ & 1.5 & 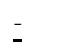 & 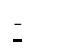 & - & - \\
\hline Parp11 & poly (ADP-ribose) polymerase family, member 11 & $\underline{101187}$ & 1 & 1.4 & - & - & - & - \\
\hline$\underline{\text { Slfn8 }}$ & $\underline{\text { schlafen } 8}$ & $\underline{276950}$ & 1.2 & 1.5 & $=$ & - & - & - \\
\hline \multicolumn{9}{|l|}{ Other } \\
\hline Oas1a & $2^{\prime}-5^{\prime}$ oligoadenylate synthetase $1 \mathrm{~A}$ & 246730 & 1.5 & 2.7 & 1.6 & 1.4 & - & - \\
\hline Oas1g & 2'-5' oligoadenylate synthetase $1 \mathrm{G}$ & 23960 & 2.3 & 3.9 & 2.5 & 2.3 & - & - \\
\hline Oas2 & $2^{\prime}-5^{\prime}$ oligoadenylate synthetase 2 & 246728 & 2.1 & 3.4 & 2 & 1.6 & - & - \\
\hline Oas3 & 2'-5' oligoadenylate synthetase 3 & 246727 & 1.1 & 2.6 & 1.3 & 1 & - & - \\
\hline Oasl1 & 2'-5' oligoadenylate synthetase-like 1 & 231655 & 2.6 & 3.5 & 2.4 & 2.5 & - & - \\
\hline Oasl2 & $2^{\prime}-5^{\prime}$ oligoadenylate synthetase-like 2 & 23962 & 2 & 3.4 & 2 & 2 & - & - \\
\hline Amica1 & adhesion molecule, interacts with CXADR antigen 1 & 270152 & - & 1.1 & - & 1.1 & - & - \\
\hline Angpt/4 & angiopoietin-like 4 & 57875 & 1.1 & 1.5 & - & 1.6 & 1.1 & - \\
\hline Asb13 & ankyrin repeat and SOCS box-containing 13 & 142688 & 1.2 & 1 & 1.1 & 1.3 & - & - \\
\hline Anxa4 & annexin A4 & 11746 & 1.8 & 1.9 & 1.7 & 2.1 & 1.1 & - \\
\hline Apol9b & apolipoprotein $L 9 b$ & 71898 & 2.3 & 2.7 & 2.6 & 2.4 & - & - \\
\hline Bst2 & bone marrow stromal cell antigen 2 & 69550 & 2.3 & 3 & 2.1 & 2.2 & - & - \\
\hline Casp4 & caspase 4, apoptosis-related cysteine peptidase & 12363 & 1.9 & 1.6 & 1.9 & 2 & - & - \\
\hline Ctsc & cathepsin C & 13032 & 1.1 & 1.1 & - & 1.1 & - & - \\
\hline $\mathrm{Cd} 274$ & CD274 antigen & 60533 & 1.9 & 2.3 & 1.6 & 2.1 & 1.2 & - \\
\hline $\mathrm{Cd} 51$ & CD5 antigen-like & 11801 & 1.2 & 1.5 & 1.4 & 1.1 & - & - \\
\hline Cd69 & CD69 antigen & 12515 & 1.8 & 1.8 & 1.5 & 1.7 & - & - \\
\hline Ccl2 (MCP1) & chemokine (C-C motif) ligand 2 & 20296 & 3.5 & 3.3 & 2.9 & 3.3 & 2.8 & - \\
\hline $\mathrm{Ccl} 3(\mathrm{MIP}-1 \mathrm{a})$ & chemokine (C-C motif) ligand 3 & 20302 & 2 & 2.1 & 2.3 & 2 & 1.4 & - \\
\hline $\mathrm{Ccl} 7$ & chemokine (C-C motif) ligand 7 & 20306 & 3 & 2.9 & 2.6 & 2.9 & 2.7 & 1.5 \\
\hline
\end{tabular}


Table 2 Selection of up-regulated genes in mouse spleens in response to MVA, LSDV, CNPV, FWPV, PEPV and FeP2. Differences in $\log _{2}$ Fold Changes (between each virus and the control) are depicted. (Continued)

\begin{tabular}{|c|c|c|c|c|c|c|c|c|}
\hline Ccr5 & chemokine (C-C motif) receptor 5 & 12774 & 1.1 & 1.5 & 1.3 & 1.3 & - & - \\
\hline $\mathrm{Ccrl} 2$ & chemokine (C-C motif) receptor-like 2 & 54199 & - & 1.5 & 1.2 & 1.4 & - & - \\
\hline Cxcl10 (IP-10) & chemokine ( $\mathrm{C}-\mathrm{X}-\mathrm{C}$ motif) ligand 10 & 15945 & 2.7 & 3 & 2.2 & 2.8 & 1.7 & - \\
\hline CxCl11 (I-TAC) & chemokine (C-X-C motif) ligand 11 & 56066 & 4.5 & 4.4 & 3.4 & 4.3 & 1.5 & - \\
\hline Cxcl9 (MIG) & chemokine (C-X-C motif) ligand 9 & 17329 & 2.1 & 2.3 & - & 1.8 & - & - \\
\hline Chi3l3 & chitinase 3-like 3 & 12655 & - & 1.2 & 1.4 & 1.6 & - & - \\
\hline Csf2rb2 & $\begin{array}{l}\text { colony stimulating factor } 2 \text { receptor, beta 2, low-affinity } \\
\text { (granulocyte-macrophage) }\end{array}$ & 12984 & - & 1.5 & - & 1.7 & - & - \\
\hline C1qa & complement component 1, q subcomponent, alpha polypeptide & 12259 & - & 1.1 & 1 & - & - & - \\
\hline $\mathrm{C} 2$ & complement component 2 (within $\mathrm{H}-2 \mathrm{~S}$ ) & 12263 & 1.5 & 1.8 & 1.7 & 1.6 & 1 & - \\
\hline $\mathrm{Cfb}$ & complement factor B & 14962 & 2.2 & 2.6 & 1.6 & 2 & 1 & - \\
\hline Cdkn1a (P21) & cyclin-dependent kinase inhibitor $1 \mathrm{~A}$ & 12575 & 1.7 & 2 & 1.5 & 2 & 1.2 & - \\
\hline Cstb & cystatin B & 13014 & 1.4 & 1 & 1.5 & 1.5 & - & - \\
\hline Cst7 & cystatin F (leukocystatin) & 13011 & 1.2 & 1.4 & 1.1 & 1.4 & - & - \\
\hline Cmpk2 & cytidine monophosphate (UMP-CMP) kinase 2, mitochondrial & 22169 & 1.3 & 2 & 1.3 & 1.3 & - & - \\
\hline Cycs & cytochrome c, somatic & 13063 & - & 1.1 & - & 1.1 & - & - \\
\hline Ctla2a & cytotoxic T lymphocyte-associated protein 2 alpha & 13024 & 1.1 & 1 & 1.1 & 1.3 & - & 1.4 \\
\hline $\mathrm{Dd} \times 60$ & DEAD (Asp-Glu-Ala-Asp) box polypeptide 60 & 234311 & 1.7 & 2.6 & 1.5 & 1.4 & - & - \\
\hline Dh×58 (LGP2) & DEXH (Asp-Glu-X-His) box polypeptide 58 & 80861 & 1.7 & 2.5 & 1.6 & 1.5 & - & - \\
\hline Dram1 & DNA-damage regulated autophagy modulator 1 & 71712 & 1.2 & 1.4 & - & 1.3 & - & - \\
\hline Ddit4 & DNA-damage-inducible transcript 4 & 74747 & 1.2 & 1.2 & 1.8 & 1.3 & 1.4 & - \\
\hline Eif2ak2 (PKR) & eukaryotic translation initiation factor 2-alpha kinase 2 & 19106 & 1.1 & 2.1 & 1.1 & 1.1 & - & - \\
\hline Daxx & Fas death domain-associated protein & 13163 & 2 & 2.3 & 1.7 & 1.9 & - & - \\
\hline Fcgr1 & Fc receptor, IgG, high affinity I & 14129 & 2.4 & 2.6 & 2.4 & 2.6 & 1.4 & - \\
\hline Fcgr4 & Fc receptor, lgG, low affinity IV & 246256 & 2.5 & 3.6 & 2.8 & 2.9 & 1.8 & - \\
\hline Fpr1 & formyl peptide receptor 1 & 14293 & 1.3 & 1.1 & 1.4 & 1.4 & - & - \\
\hline Fpr2 & formyl peptide receptor 2 & 14289 & 1.8 & 1.5 & 1.7 & 1.9 & - & - \\
\hline Glipr2 & GLI pathogenesis-related 2 & 384009 & 1.4 & 1.5 & 1.1 & 1.5 & - & - \\
\hline Gp49a & glycoprotein $49 \mathrm{~A}$ & 14727 & 2.6 & 2.4 & 2.7 & 2.7 & 1.9 & 2 \\
\hline Gca & grancalcin & 227960 & 1.2 & 1.3 & 1.4 & 1.6 & - & - \\
\hline Gzma & granzyme A & 14938 & 1.8 & 1.8 & 2.2 & 2 & - & - \\
\hline Gzmb & granzyme B & 14939 & 3.7 & 4.7 & 4.1 & 4.2 & 2.4 & - \\
\hline Gadd45b & growth arrest and DNA-damage-inducible 45 beta & 17873 & 1.3 & 1.5 & 1.2 & 1.5 & - & - \\
\hline Gbp1 & guanylate binding protein 1 & 14468 & 2.1 & 2.7 & 1.6 & 2.5 & - & - \\
\hline Gbp11 & guanylate binding protein 11 & 634650 & 3.9 & 4.5 & 2.9 & 4.2 & 1.6 & - \\
\hline Gbp2 & guanylate binding protein 2 & 14469 & 2.3 & 2.8 & 1.5 & 2.7 & - & - \\
\hline Gbp3 & guanylate binding protein 3 & 55932 & 1.6 & 1.8 & 1.2 & 1.6 & - & - \\
\hline Gbp4 & guanylate binding protein 4 & 17472 & 2.1 & 2.7 & 1.3 & 2.4 & - & - \\
\hline Gbp5 & guanylate binding protein 5 & 229898 & 2 & 2.8 & 1.3 & 2.2 & 1.1 & - \\
\hline Gbp7 & guanylate binding protein 7 & 229900 & 1.6 & 2.2 & 1.2 & 1.7 & - & - \\
\hline Gbp10 & guanylate-binding protein 10 & 626578 & 2.1 & 3.2 & 1.3 & 1.9 & - & - \\
\hline Gbp8 & guanylate-binding protein 8 & 76074 & 1.3 & 1.4 & 1.4 & 2.1 & - & - \\
\hline Gbp9 & guanylate-binding protein 9 & 236573 & 1.2 & 1.9 & 1 & 1.3 & - & - \\
\hline $\mathrm{Hp}$ & haptoglobin & 15439 & 1.2 & 1.8 & 1.7 & 1.9 & 1.3 & 1.3 \\
\hline
\end{tabular}


Table 2 Selection of up-regulated genes in mouse spleens in response to MVA, LSDV, CNPV, FWPV, PEPV and FeP2. Differences in $\log _{2}$ Fold Changes (between each virus and the control) are depicted. (Continued)

\begin{tabular}{|c|c|c|c|c|c|c|c|c|}
\hline Hspa1b & heat shock protein $1 \mathrm{~B}$ & 15511 & 2.3 & 2.9 & 2.4 & 2.5 & - & - \\
\hline $\mathrm{H} 2-\mathrm{Q} 4$ & histocompatibility 2, Q region locus 4 & 15015 & 1 & 1.2 & - & 1.2 & - & - \\
\hline H2-Q6 & histocompatibility 2, Q region locus 6 & 110557 & 1 & 1 & - & 1.1 & - & - \\
\hline $\mathrm{H} 2-\mathrm{T} 22$ & histocompatibility 2, T region locus 22 & 15039 & 1 & 1.3 & - & - & - & - \\
\hline $\mathrm{H} 2-\mathrm{T} 23$ & histocompatibility 2, T region locus 23 & 15040 & 1 & 1.3 & - & 1 & - & - \\
\hline $\operatorname{lrgm} 1$ & immunity-related GTPase family M member 1 & 15944 & 1.4 & 2.3 & 1.1 & 1.4 & - & - \\
\hline $\operatorname{lrgm} 2$ & immunity-related GTPase family M member 2 & 54396 & 1 & 1.7 & - & 1.2 & - & - \\
\hline $\operatorname{lrg} 1$ & immunoresponsive gene 1 & 16365 & 2.6 & 2.5 & 1.9 & 2.5 & 1.6 & - \\
\hline Ifi202b & interferon activated gene $202 \mathrm{~B}$ & 26388 & 2.4 & 2.6 & 2.1 & 2.2 & - & - \\
\hline Ifi204 & interferon activated gene 204 & 15951 & 3.3 & 4 & 3.2 & 3.9 & - & - \\
\hline lgtp & interferon gamma induced GTPase & 16145 & 1.4 & 2.2 & 1 & 1.8 & - & - \\
\hline Ifitm6 & interferon induced transmembrane protein 6 & 213002 & 1.8 & 1.9 & 2.4 & 2.3 & 1.6 & 1.5 \\
\hline Ifih1 (MDA5) & interferon induced with helicase $C$ domain 1 & 71586 & 1.3 & 2.1 & 1.2 & 1.2 & - & - \\
\hline ligp1 & interferon inducible GTPase 1 & 60440 & 1.7 & 2.9 & 1.2 & 1.9 & - & - \\
\hline Irf1 & interferon regulatory factor 1 & 16362 & - & 1.3 & - & 1.1 & - & - \\
\hline Irf7 & interferon regulatory factor 7 & 54123 & 1.7 & 2.9 & 1.7 & 1.1 & - & - \\
\hline Ifi35 & interferon-induced protein 35 & 70110 & 1.1 & 1.5 & 1.1 & 1.2 & - & - \\
\hline Ifi44 & interferon-induced protein 44 & 99899 & 2 & 2.5 & 1.8 & 1.6 & - & - \\
\hline |fi44| & interferon-induced protein 44 like & 15061 & 2.1 & 2.9 & 2 & 2 & - & - \\
\hline Ifit1 & interferon-induced protein with tetratricopeptide repeats 1 & 15957 & 2.2 & 3.3 & 2 & 1.7 & - & - \\
\hline Ifit2 & interferon-induced protein with tetratricopeptide repeats 2 & 15958 & 1.8 & 2.5 & 1.8 & 1.7 & - & - \\
\hline$\| 1 \mathrm{a}$ & interleukin 1 alpha & 16175 & 1.9 & 2 & 2.2 & 2.3 & 1.8 & - \\
\hline$\| 1 f 9$ & interleukin 1 family, member 9 & 215257 & 1.3 & 1.6 & 1.7 & 1.8 & 1.2 & 1 \\
\hline$\| 1 \mathrm{rn}$ & interleukin 1 receptor antagonist & 16181 & 1.3 & 1.1 & - & 1.2 & - & - \\
\hline$\| 12 \mathrm{rb} 1$ & interleukin 12 receptor, beta 1 & 16161 & 2 & 2.4 & 1.7 & 2.1 & 1.1 & - \\
\hline$\| 12 \mathrm{rb} 2$ & interleukin 12 receptor, beta 2 & 16162 & 1.2 & 1.3 & 1.3 & 1.3 & - & - \\
\hline$\| 15$ & interleukin 15 & 16168 & 1.1 & 1.3 & 1.2 & 1 & - & - \\
\hline Il15ra & interleukin 15 receptor, alpha chain & 16169 & 1.8 & 1.9 & 1.5 & 1.8 & 1.2 & - \\
\hline$\| 18 b p$ & interleukin 18 binding protein & 16068 & 1.2 & 1.7 & 1.3 & 1.5 & - & - \\
\hline$\| 2$ ra & interleukin 2 receptor, alpha chain & 16184 & 1.1 & 1.2 & - & 1.3 & 1.1 & - \\
\hline$\| 33$ & interleukin 33 & 77125 & - & 1 & - & 1.1 & - & - \\
\hline $\operatorname{lsg} 15$ & ISG15 ubiquitin-like modifier & 100038882 & 1.6 & 2.2 & 1.5 & 1.4 & - & - \\
\hline Klrk1 & killer cell lectin-like receptor subfamily K, member 1 & 27007 & 1.5 & 1.7 & 1.6 & 1.5 & - & - \\
\hline Lgals9 & lectin, galactose binding, soluble 9 & 16859 & 1.2 & 1.6 & 1.1 & 1.2 & - & - \\
\hline Lgals3bp & lectin, galactoside-binding, soluble, 3 binding protein & 19039 & 1.2 & 1.7 & 1.1 & - & - & - \\
\hline Lilrb4 & leukocyte immunoglobulin-like receptor, subfamily B, member 4 & 14728 & 1.6 & 1.5 & 1.7 & 1.7 & 1.1 & - \\
\hline $\operatorname{Lcn} 2$ & lipocalin 2 & 16819 & 1.4 & 1.6 & 2.2 & 2.2 & 1.4 & 1.7 \\
\hline Ly6a & lymphocyte antigen 6 complex, locus A & 110454 & 1.7 & 1.9 & 1.2 & 1.3 & - & - \\
\hline Ly6c1 & lymphocyte antigen 6 complex, locus $\mathrm{C} 1$ & 17067 & 1.8 & 2.2 & 1.9 & 1.9 & - & - \\
\hline Ly6c2 & lymphocyte antigen 6 complex, locus C2 & 100041546 & 1.3 & 1.6 & 1.3 & 1.1 & - & - \\
\hline Ly6g & lymphocyte antigen 6 complex, locus $G$ & 546644 & - & 1.7 & 2.5 & 2.2 & - & - \\
\hline Msr1 & macrophage scavenger receptor 1 & 20288 & 2.3 & 2 & 2 & 2.2 & 1.4 & 1.1 \\
\hline Mmp13 & matrix metallopeptidase 13 & 17386 & 2.7 & 2.6 & 2.4 & 2.4 & 1.5 & - \\
\hline Mmp19 & matrix metallopeptidase 19 & 58223 & 1.9 & 2 & 2 & 2 & 1.5 & 1.2 \\
\hline
\end{tabular}


Table 2 Selection of up-regulated genes in mouse spleens in response to MVA, LSDV, CNPV, FWPV, PEPV and FeP2. Differences in $\log _{2}$ Fold Changes (between each virus and the control) are depicted. (Continued)

\begin{tabular}{|c|c|c|c|c|c|c|c|c|}
\hline Mmp25 & matrix metallopeptidase 25 & 240047 & - & 1 & - & 1.1 & - & - \\
\hline Mmp8 & matrix metallopeptidase 8 & 17394 & 2.7 & 3 & 3.1 & 3.4 & 2.5 & 2.8 \\
\hline Ms4a4a & membrane-spanning 4-domains, subfamily A, member 4A & 666907 & 2.6 & 2.7 & 2.6 & 2.5 & 1.5 & 1.1 \\
\hline Ms4a4c & membrane-spanning 4-domains, subfamily A, member $4 C$ & 64380 & 1.2 & 1.5 & 1.2 & 1 & - & - \\
\hline Ms4a4d & membrane-spanning 4-domains, subfamily A, member 4D & 66607 & 1.6 & 1.5 & 1.6 & 1.8 & - & - \\
\hline Ms4a6c & membrane-spanning 4-domains, subfamily A, member $6 \mathrm{C}$ & 73656 & 1.1 & 1.1 & 1.2 & 1.2 & - & - \\
\hline Ms4a6d & membrane-spanning 4-domains, subfamily A, member 6D & 68774 & 3.2 & 3.5 & 3.1 & 3.3 & - & - \\
\hline Ms4a7 & membrane-spanning 4-domains, subfamily A, member 7 & 109225 & 1.7 & 1.4 & 2 & 1.9 & 1.4 & - \\
\hline Mlkl & mixed lineage kinase domain-like & 74568 & 2.1 & 2.7 & 2 & 2.3 & - & - \\
\hline Mnda & myeloid cell nuclear differentiation antigen & 381308 & 1.8 & 1.8 & 1.6 & 1.7 & - & - \\
\hline Mndal & myeloid nuclear differentiation antigen like & $1 E+08$ & 1.1 & 1.3 & 1.1 & 1.2 & - & - \\
\hline Mx1 & myxovirus (influenza virus) resistance 1 & 17857 & 3.2 & 3.9 & 3 & 2.8 & - & - \\
\hline$M \times 2$ & myxovirus (influenza virus) resistance 2 & 17858 & 2.7 & 3.7 & 2.1 & 2.2 & - & - \\
\hline Nampt & nicotinamide phosphoribosyltransferase & 59027 & 1.7 & 1.9 & 1.5 & 2 & - & - \\
\hline $\mathrm{Nmi}$ & $\mathrm{N}$-myc (and STAT) interactor & 64685 & 1.3 & 1.6 & 1.1 & 1.5 & - & - \\
\hline Prf1 & perforin 1 (pore forming protein) & 18646 & 1.2 & 1.4 & 1.1 & 1.2 & - & - \\
\hline Phf11a & PHD finger protein $11 \mathrm{~A}$ & 219131 & 1.2 & 1.4 & 1.1 & 1.1 & - & - \\
\hline Phf11b & PHD finger protein 11B & 236451 & 2 & 1.8 & 1.7 & 1.8 & - & - \\
\hline Phf11c & PHD finger protein $11 \mathrm{C}$ & 628705 & 2 & 2.4 & 1.7 & 1.7 & - & - \\
\hline Phf11d & PHD finger protein 11D & 219132 & 2.4 & 2.9 & 2.4 & 2.4 & 1.1 & - \\
\hline Plac8 & placenta-specific 8 & 231507 & 1.3 & 1.7 & - & 1.3 & - & - \\
\hline Parp10 & poly (ADP-ribose) polymerase family, member 10 & 671535 & 1.2 & 1.5 & - & 1.2 & - & - \\
\hline Parp12 & poly (ADP-ribose) polymerase family, member 12 & 243771 & 1.5 & 2.5 & 1.3 & 1.6 & - & - \\
\hline Parp14 & poly (ADP-ribose) polymerase family, member 14 & 547253 & 1.1 & 1.7 & - & 1 & - & - \\
\hline Parp9 & poly (ADP-ribose) polymerase family, member 9 & 80285 & 1.3 & 1.9 & 1.1 & 1.2 & - & - \\
\hline Psme1 & proteasome (prosome, macropain) 28 subunit, alpha & 19186 & - & 1.1 & - & 1 & - & - \\
\hline Psma7 & proteasome (prosome, macropain) subunit, alpha type 7 & 26444 & - & 1.1 & - & 1.1 & - & - \\
\hline Psmb10 & proteasome (prosome, macropain) subunit, beta type 10 & 19171 & 1.1 & 1.4 & - & 1.3 & - & - \\
\hline Psmb8 & $\begin{array}{l}\text { proteasome (prosome, macropain) subunit, beta type } 8 \\
\text { (large multifunctional peptidase } 7 \text { ) }\end{array}$ & 16913 & - & 1.4 & - & 1.1 & - & - \\
\hline Pyhin1 & pyrin and HIN domain family, member 1 & 236312 & 1.7 & 1.8 & 1.5 & 1.4 & - & - \\
\hline Pydc3 & pyrin domain containing 3 & 100033459 & 1.9 & 2.4 & 1.7 & 1.5 & - & - \\
\hline Pydc4 & pyrin domain containing 4 & 623121 & 2.9 & 3.4 & 2.2 & 1.9 & - & - \\
\hline Ppa1 & pyrophosphatase (inorganic) 1 & 67895 & 1.3 & 2.1 & - & 1.5 & - & - \\
\hline Pdk4 & pyruvate dehydrogenase kinase, isoenzyme 4 & 27273 & 1.5 & 1.1 & 1.7 & 1.4 & 1.8 & 1.8 \\
\hline Rtp4 & receptor transporter protein 4 & 67775 & 1.4 & 2.2 & 1.2 & 1.1 & - & - \\
\hline Retnlg & resistin like gamma & 245195 & 1.1 & 1.3 & 1.5 & 1.6 & 1.4 & 1.6 \\
\hline Rnf19b & ring finger protein 19B & 75234 & - & 1.2 & - & 1.1 & - & - \\
\hline Rnf213 & ring finger protein 213 & 672511 & 1.4 & 2.2 & 1 & 1 & - & - \\
\hline Slfn1 & schlafen 1 & 20555 & 1.8 & 1.9 & 1.3 & 1.5 & - & - \\
\hline Slfn3 & schlafen 3 & 20557 & 1.3 & 1.7 & 1.5 & 1.7 & - & - \\
\hline Slfn4 & schlafen 4 & 20558 & 1.9 & 3 & 2 & 1.8 & - & - \\
\hline Slfn5 & schlafen 5 & 327978 & 1.5 & 2.4 & 1.4 & 1.1 & - & - \\
\hline Slfng & schlafen 9 & 237886 & 1.5 & 2.4 & 1.4 & 1.7 & - & - \\
\hline
\end{tabular}


Table 2 Selection of up-regulated genes in mouse spleens in response to MVA, LSDV, CNPV, FWPV, PEPV and FeP2. Differences in $\log _{2}$ Fold Changes (between each virus and the control) are depicted. (Continued)

\begin{tabular}{|c|c|c|c|c|c|c|c|c|}
\hline Serpina3f & serine (or cysteine) peptidase inhibitor, clade A, member $3 \mathrm{~F}$ & 238393 & 1.9 & 2.7 & 1.3 & 2.2 & 1.3 & - \\
\hline Serpinb9 & serine (or cysteine) peptidase inhibitor, clade B, member 9 & 20723 & 1.3 & 1.1 & 1.1 & 1.4 & - & - \\
\hline Serpinb9b & serine (or cysteine) peptidase inhibitor, clade $B$, member $9 \mathrm{~b}$ & 20706 & 1.2 & 1 & 1.1 & - & - & - \\
\hline Serpine1 & serine (or cysteine) peptidase inhibitor, clade $E$, member 1 & 18787 & 1.1 & - & - & 1 & - & - \\
\hline Stat2 & signal transducer and activator of transcription 2 & 20847 & 1.4 & 1.9 & 1.2 & 1.5 & - & - \\
\hline Slamf8 & SLAM family member 8 & 74748 & - & 1.1 & - & 1.2 & - & - \\
\hline Slc15a3 & solute carrier family 15, member 3 & 65221 & - & 1.3 & - & 1.1 & - & - \\
\hline Slc25a22 & solute carrier family 25 (mitochondrial carrier, glutamate), member 22 & 68267 & - & 1.2 & 1 & 1.2 & - & - \\
\hline Socs1 & suppressor of cytokine signaling 1 & 12703 & 1.6 & 2.2 & - & 1.9 & - & - \\
\hline Socs 2 & suppressor of cytokine signaling 2 & 216233 & 1 & 1.4 & - & 1.8 & - & - \\
\hline Tgtp1 & T cell specific GTPase 1 & 21822 & - & 1.4 & - & 1.1 & - & - \\
\hline Tgtp2 & T cell specific GTPase 2 & $1.00 \mathrm{E}+08$ & 1.6 & 2.7 & - & 1.5 & - & - \\
\hline Trex1 & three prime repair exonuclease 1 & 22040 & - & 1.2 & - & 1 & - & - \\
\hline Timp1 & tissue inhibitor of metalloproteinase 1 & 21857 & 2.8 & 2.9 & 2.4 & 2.9 & 1.8 & 1.6 \\
\hline $\operatorname{Tlr} 13$ & toll-like receptor 13 & 279572 & 1.4 & 1 & 1.7 & 1.5 & 1 & - \\
\hline T/r3 & toll-like receptor 3 & 142980 & 1 & 1.4 & 1.2 & 1.1 & - & - \\
\hline Tlr7 & toll-like receptor 7 & 170743 & 1.1 & 1.2 & 1.2 & - & - & - \\
\hline Tlr8 & toll-like receptor 8 & 170744 & 1.1 & 1.1 & 1.3 & 1 & - & - \\
\hline Trafd1 & TRAF type zinc finger domain containing 1 & 231712 & 1.1 & 1.6 & 1 & 1.1 & - & - \\
\hline Trem3 & triggering receptor expressed on myeloid cells 3 & 58218 & 1.1 & 1.4 & 1.2 & 1.5 & - & - \\
\hline Trim12c & tripartite motif-containing $12 \mathrm{C}$ & 319236 & - & 1.5 & 1.2 & 1.3 & - & - \\
\hline Trim21 & tripartite motif-containing 21 & 20821 & 1.1 & 1.3 & - & 1.4 & - & - \\
\hline Trim30a & tripartite motif-containing $30 \mathrm{~A}$ & 20128 & 1.3 & 2.1 & - & 1.1 & - & - \\
\hline Trim30c & tripartite motif-containing $30 \mathrm{C}$ & 434219 & 2.6 & 3.4 & 2.5 & 2.2 & - & - \\
\hline Trim30d & tripartite motif-containing 30D & 209387 & 3.1 & 3.5 & 3.4 & 2.7 & 1.4 & - \\
\hline Wars & tryptophanyl-tRNA synthetase & 22375 & 1.1 & 1.6 & - & 1.4 & - & - \\
\hline Tnfsf10 & tumor necrosis factor (ligand) superfamily, member 10 & 22035 & 2 & 2.3 & 2 & 1.9 & - & - \\
\hline Usp18 & ubiquitin specific peptidase 18 & 24110 & 2.2 & 3.1 & 2 & 1.8 & - & - \\
\hline Zbp1 & Z-DNA binding protein 1 & 58203 & 1.9 & 2.7 & 1.3 & 1.6 & - & - \\
\hline
\end{tabular}

Italics: Genes induced by LSDV alone

Italics and underlined: Genes induced by CNPV, FWPV and MVA only, and not LSDV

Bold: Genes induced by Avipoxviruses only (CNPV, FWPV, FeP2 and/ or PEPV)

Bold and Italics: Genes induced by FWPV only

Bold and underlined: Genes induced by CNPV only

Underlined: Genes induced by LSDV and MVA, but not by the Avipoxviruses

transcriptomes were identical then it was unlikely that they would differ as vaccine vectors. The complex model of the mouse spleen was selected because the spleen is rich in immune cells and the immune response is a complex interaction between different types of cells and their proteins which would not be reflected in in vitro models. Although in vitro expression studies have provided useful information, gene expression profiles performed in cell culture may not accurately reflect the changes that occur as result of infection in vivo. It is anticipated that different poxviruses will have different properties that will relate to their ability to act as adjuvants driving the immune response to the vaccine protein towards a particular type of immune response.

Unsupervised hierarchical clustering differentiates between the observed responses to the six poxviruses, grouping CNPV and MVA together and FWPV in a separate cluster (more closely related to CNPV and MVA than to LSDV) (Fig. 1). FeP2 and PEPV group together in a cluster that is separate from the other four viruses (Fig. 1). This grouping is quite different from phylogenetic relationships established by DNA sequence comparisons [27]. It is also not dependent on the viral morphogenesis 
Table 3 Selection of down-regulated genes in mouse spleens in response to MVA, LSDV, CNPV, FWPV, PEPV and FeP2. Differences in $\log _{2}$ Fold Changes (between each virus and the control) are depicted

\begin{tabular}{|c|c|c|c|c|c|c|c|c|}
\hline Symbol & Name & Entrez & MVA & LSDV & CNPV & FWPV & PEPV & $\mathrm{FeP2}$ \\
\hline \multicolumn{9}{|c|}{ Genes down-regulated by LSDV ALONE } \\
\hline Adamdec1 & ADAM-like, decysin 1 & 58860 & - & -1.1 & - & - & - & - \\
\hline$C d 59 a$ & CD59a antigen & 12509 & - & -1.2 & - & - & - & - \\
\hline$C d 7$ & CD7 antigen & 12516 & - & -1.1 & - & - & - & - \\
\hline Esm 1 & endothelial cell-specific molecule 1 & 71690 & - & -1.1 & - & - & - & - \\
\hline lgfbp3 & insulin-like growth factor binding protein 3 & 16009 & - & -1 & - & - & - & - \\
\hline lgk & immunoglobulin kappa chain complex & 243469 & - & -1 & - & - & - & - \\
\hline Lilra5 & leukocyte immunoglobulin-like receptor, subfamily A (with TM domain), member 5 & 232801 & - & -1.1 & - & - & - & - \\
\hline Prkcg & protein kinase C, gamma & 18752 & - & -1 & - & - & - & - \\
\hline \multicolumn{9}{|c|}{ Genes down-regulated by MVA alone } \\
\hline Ctsf & cathepsin F & 56464 & -1.1 & - & - & - & - & - \\
\hline Depdc1a & DEP domain containing $1 \mathrm{a}$ & 76131 & -1.1 & - & - & - & - & - \\
\hline Diap3 & diaphanous homolog 3 (Drosophila) & 56419 & -1.1 & - & - & - & - & - \\
\hline Hmmr (CD168) & hyaluronan mediated motility receptor (RHAMM) & 15366 & -1 & - & - & - & - & - \\
\hline TIr11 & toll-like receptor 11 & 239081 & -1 & - & - & - & - & - \\
\hline \multicolumn{9}{|c|}{ Genes down-regulated by Isdv and MVA, BUT NOT BY CNPV and FWPV } \\
\hline$\underline{\mathrm{Ccl} 21 \mathrm{a}}$ & chemokine (C-C motif) ligand 21 A (serine) & $\underline{18829}$ & $\underline{-1.3}$ & -1.7 & - & - & - & - \\
\hline$\underline{\mathrm{Ccl} 21 \mathrm{~b}}$ & chemokine (C-C motif) ligand 21B (leucine) & 100042493 & $\underline{-1.2}$ & $\underline{-1.7}$ & - & - & - & - \\
\hline$\underline{\mathrm{Ccl} 21 \mathrm{c}}$ & chemokine (C-C motif) ligand 21C (leucine) & $\underline{65956}$ & $\underline{-1.2}$ & $\underline{-1.6}$ & - & - & - & - \\
\hline$\underline{\text { Kel }}$ & Kell blood group & 23925 & $\underline{-1.5}$ & $\underline{-1.5}$ & - & - & - & - \\
\hline$\underline{\text { Slc12a2 }}$ & $\underline{\text { solute carrier family } 12, \text { member } 2}$ & $\underline{20496}$ & -1 & -1 & $=$ & $=$ & $=$ & - \\
\hline$\underline{\text { Timd4 }}$ & $\underline{\text { T cell immunoglobulin and mucin domain containing } 4}$ & $\underline{276891}$ & $\underline{-1.1}$ & $\underline{-1.3}$ & $\overline{-}$ & $=$ & 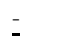 & $=$ \\
\hline \multicolumn{9}{|c|}{ Genes down-regulated by CNPV, FWPV AND MVA ONLY, and not LSDV. } \\
\hline$\underline{T s p a n 33}$ & tetraspanin 33 & $\underline{232670}$ & $\underline{-1.6}$ & 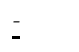 & $\underline{-1.3}$ & $\underline{-1.1}$ & $=$ & $=$ \\
\hline \multicolumn{9}{|l|}{ OTHER } \\
\hline Abca9 & ATP-binding cassette, sub-family A (ABC1), member 9 & 217262 & -1.3 & -1.5 & - & -1.1 & - & - \\
\hline Aplnr & apelin receptor & 23796 & -1.4 & -1.5 & -1.2 & -1.2 & - & - \\
\hline Cd209a & CD209a antigen & 170786 & -1.7 & -2.2 & -1.1 & -1.5 & - & - \\
\hline Cd209b & CD209b antigen & 69165 & -1.2 & -1.3 & - & - & - & - \\
\hline Cldn13 & claudin 13 & 57255 & -1.5 & -1.2 & -1.1 & - & - & - \\
\hline Emr4 & EGF-like module containing, mucin-like, hormone receptor-like sequence 4 & 52614 & -1.4 & -1.8 & -1.1 & -1.5 & -1.3 & - \\
\hline Fcamr & Fc receptor, $\lg A$, IgM, high affinity & 64435 & -1.1 & - & -1.5 & - & - & - \\
\hline Fcer2a & Fc receptor, IgE, low affinity II, alpha polypeptide & 14128 & -2.6 & -3 & -2.4 & -2.5 & -2.2 & -1.3 \\
\hline H2-M2 & histocompatibility 2, M region locus 2 & 14990 & -1 & -1.4 & -1 & -1 & -1.1 & - \\
\hline Hs3st2 & heparan sulfate (glucosamine) 3-O-sulfotransferase 2 & 195646 & -1.4 & -1.3 & -1 & -1 & - & - \\
\hline Ifi27l1 & interferon, alpha-inducible protein 27 like 1 & 52668 & -1.4 & -1.3 & - & - & - & - \\
\hline Mgst3 & microsomal glutathione S-transferase 3 & 66447 & -1.6 & -1 & - & -1 & - & - \\
\hline Slc16a10 & solute carrier family 16 (monocarboxylic acid transporters), member 10 & 72472 & -1.4 & - & - & -1.1 & - & - \\
\hline Slc2a4 & solute carrier family 2 (facilitated glucose transporter), member 4 & 20528 & -1.7 & -1 & - & -1.1 & - & - \\
\hline Slc38a5 & solute carrier family 38 , member 5 & 209837 & -1.4 & -1.2 & -1 & - & - & - \\
\hline Slc6a20a & solute carrier family 6 (neurotransmitter transporter), member 20A & 102680 & -1.4 & -1.3 & -1 & -1 & - & - \\
\hline Tfrc & transferrin receptor & 22042 & -1.2 & - & - & - & - & - \\
\hline Tspan8 & tetraspanin 8 & 216350 & -1.4 & - & - & -1 & - & - \\
\hline
\end{tabular}

Italics genes down-regulated by LSDV alone

Bold Genes down-regulated by MVA alone

Underlined genes down-regulated by LSDV and MVA, but not by avipoxviruses

Italics and underlined genes down-regulated by CNPV, FWPV and MVA only, and not LSDV 
in non-permissive cells. PEPV and FWPV have been demonstrated to infect mammalian cells [28] and progress to a late stage in morphogenesis $[28,49]$ whereas FeP2 [50] and CNPV have a block prior to DNA replication [51]. It is noted that infectivity studies have not been done in mouse spleens and that this may be different to published data on other mammalian cells. A further study is needed to determine if PEPV and FeP2 infect the same number of cells in the mouse spleen as the other viruses.

Amongst the four avipoxviruses analysed here, the greatest difference in host responses was expected between CNPV and FWPV, as on a genomic level, these viruses are significantly divergent with amino acid identity between ORF homologues (55-74\%) being similar to that observed between different ChPV genera [52]. We did not expect to see such significant differences between the host responses induced by $\mathrm{FeP} 2$ and PEPV which share 94.4\% nucleotide identity with each other and 85.3 and $84.0 \%$ nucleotide identity with FWPV respectively [27]. Since avipoxviruses are restricted to avian hosts, one would anticipate fewer differences between the mammalian host responses induced by them as it is highly likely that their proteins are not as functional in mammalian cells as those of MVA and LSDV. However we show that three relatively closely related avipoxviruses (FWPV, FeP2 and PEPV) induce significant differences in gene expression in the host. FWPV induced the strongest host response in mice whereas FeP2 infection resulted in remarkably little change in host gene expression. A vaccine vector with low host reactivity, such as observed with PEPV or FeP2, might have the advantage of being a good vector to include adjuvant genes to enhance immunogenicity. Further investigation is needed to determine if there would be a good immune response to foreign antigens expressed by these vectors.

Previous in vitro studies with VACV, MVA and NYVAC have shown more host genes to be down-regulated than up-regulated [11-14]; however, this was not the case in other poxviruses, including cowpox virus (CPXV) [53], monkeypox virus (MPXV) [53] or ALVAC [15]. Unlike our study, done in a mouse model, all these studies were done in cell culture. We show that fewer genes are downregulated than up-regulated in response to in vivo infection at $24 \mathrm{~h}$ post infection (Table 1 ). For each poxvirus tested several of the dysregulated mouse genes are not yet annotated suggesting biological roles for unannotated genes and highlighting the importance of further functional analysis and annotation of the mouse genome. Contrary to in vitro studies, MVA caused more transcripts to be up-regulated than down-regulated in mouse spleens. MVA infection did, however, result in a greater number of down-regulated transcripts compared to LSDV, FWPV and CNPV.

MVA and LSDV induced the down-regulation of several genes that were not affected by the avipoxviruses tested including three forms of the chemokine CCL21 (Ccl21a, Ccl21b, Ccl21c) which are potent chemoattractants for lymphocytes and dendritic cells [40] (Table 3). VACV A41L encodes a chemokine binding protein which binds and inhibits CCL21 [54] and deletion mutants lacking the A41L gene, induce stronger virus-specific CD8+ T-cell responses $[54,55]$. LSDV does not have a homolog of the A41L gene; there must be other mechanisms that mammalian poxviruses have evolved to evade the effects of CCL21, which is clearly important for the host in clearing poxvirus infection. In selecting/designing a vaccine vector it would be desirable to use a virus which lacks A41L and does not down regulate CCL21 if a strong CD8+ T cell response is required.

Interestingly, MVA, LSDV, FWPV and CNPV all down-regulated the gene encoding the murine homo$\log$ for DC-specific ICAM-3-grabbing nonintegrin (DC SIGN) (Cd209a). Furthermore MVA, LSDV and FWPV down-regulated an additional DC SIGN homolog, CD209b (SIGNR1) (Table 3).

LSDV has a host-range restricted to ruminants and is currently being investigated as an HIV vaccine vector $[3,56]$. LSDV caused the most significant response in mice compared to the other poxviruses, both in terms of the number of up-regulated genes and the magnitude and breadth of the type I Interferon response (Fig. 4). LSDV clustered independently from the avipoxviruses and MVA. LSDV up-regulated genes are involved in the antigen processing and presentation pathway (H2-T24, Tap1 and Tap2). Furthermore, LSDV uniquely upregulated the gene encoding macrophage migration inhibitory factor (Mif), which is important in both macrophage function and T-cell immunity [57], and Ddx58, otherwise known as RIG-I (retinoic acid-inducible gene 1), which recognises viral RNA, activating downstream signalling pathways that facilitate type I IFN production [58]. The up-regulation of RIG-I may, in part, be responsible for the increased type I IFN response seen in LSDV-infected mice. Another one of the many genes uniquely up-regulated by LSDV was the transcription factor (Myc) that promotes growth, proliferation and apoptosis [59]. Myc has been shown to be up-regulated in response to infection with NYVAC and MVA in HeLa cells [13]. The absence of Myc up regulation in mouse spleens by MVA was unexpected. In a study done in Rhesus macaques rLSDV vector expressing an HIV polyprotein was immunogenic at a dose 1000-fold lower than that of rMVA. Both CD4+ and CD8+ responses were induced, rather than a predominance of $\mathrm{CD} 4+\mathrm{T}$ cells observed typically for poxvirus vectors [3].

Both LSDV and MVA up-regulated a cellular homolog of Moloney Leukemia Virus 10 (Mov10), which has been shown to inhibit retrovirus replication and infectivity [60]. It specifically interacts with the nucleocapsid 


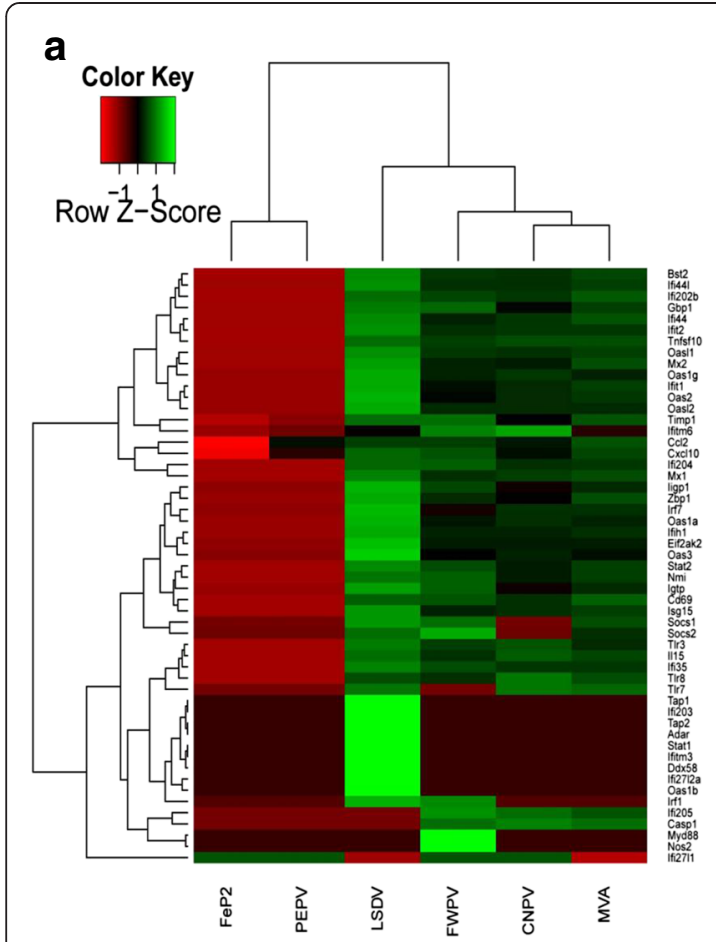

b

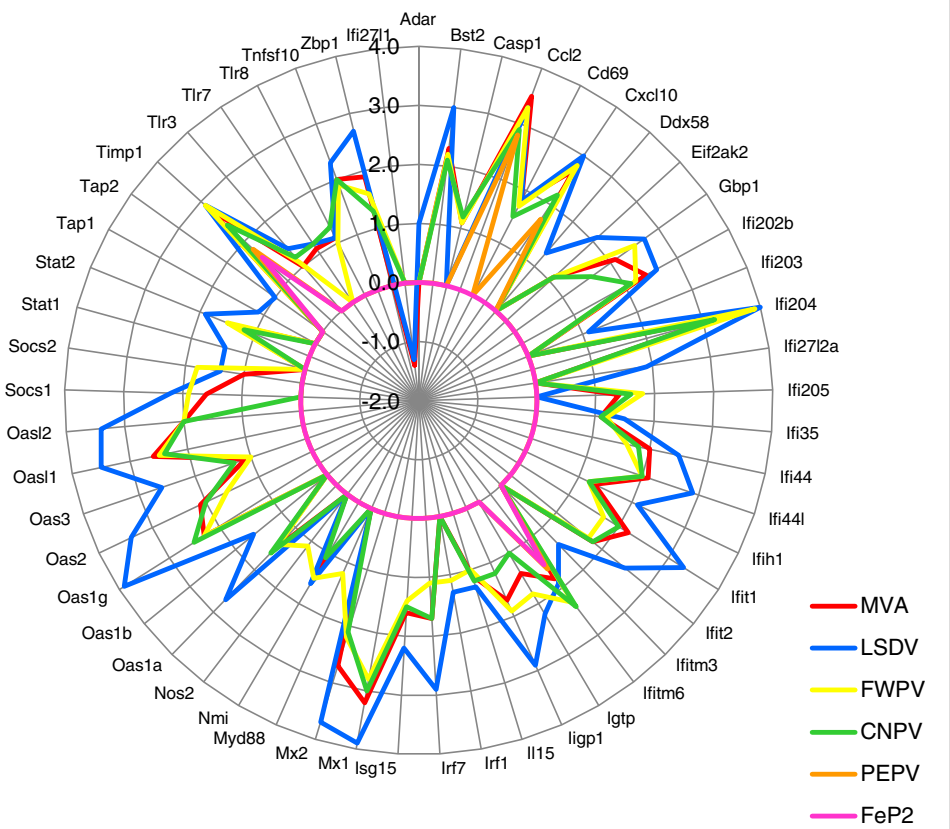

Fig. 3 Heatmap (a) and radial plot (b) depicting the differences in the type I Interferon response induced by canarypox virus (CNPV), pigeonpox (FeP2), fowlpox virus (FWPV), lumpy skin disease virus (LSDV), modified vaccinia Ankara (MVA) and penguinpox virus (PEPV). Fig. 3. a represents the $\log _{2}$ fold change $(F C)$ induction of the different genes up-regulated in the six samples compared to the control. A log 2 FC of 0 is given where genes are not present over the cut off $( \pm 1)$. Unsupervised hierarchical clustering of the samples is represented by dendograms. Clustering analysis and heatmap was performed in the R package, gplots (Warnes, 2009). Fig. 3. b shows a radial plot depicting the magnitude and breadth of the type I interferon response induced by the six viruses. The distance from the centre of the plot indicates log2-fold change (ranging from -2 to 4 )

domain of HIV Gag [60], which may have implications for vaccine vectors encoding Gag proteins. Avipoxviruses may therefore be better vectors than MVA or LSDV for the expression of Gag.

CNPV and FWPV induce the up regulation of two immunoglobulin genes (Ighg and Ighg3 (IgG3)) with CNPV up regulating a third, Ighm (Fig. 4b). All six viruses down-regulated the IgE FC receptor alpha (Fcer2a) polypeptide gene. IgE is involved in allergic responses and not vaccine responses. Antibodies of the same epitope specificity but of a different subclass can have different antibody effector functions [61]. In a recent comparison of the immune responses resulting from the partially effective clinical RV144 HIV-1 trial and the ineffective VAX003 trial, it was shown that HIV-1-specific IgG3 antibodies were correlated with decreased risk of HIV-1 infection in the RV144 trial. It is suggested that the canarypox virus, ALVAC-HIV (vCP1521) prime component of RV144 may have stimulated different antibody subclasses, specifically IgG3, compared to the protein-only vaccine (VAX003) [61]. The up regulation of IgG3 specifically by FWPV and CNPV in vivo, suggests that these two avipoxvirus vectors may be involved in stimulation of the clinically important IgG3 antibody subclass. Up regulation of IgG3 was not detected in ALVAC-infected monocyte derived dendritic cells (MDDCs) [15]; this potentially significant finding is an example of the importance of in vivo testing.

Type I IFN responses have been highlighted in previous studies investigating host gene expression changes in response to different host-restricted poxviruses [14, 15]. Type I IFN induces an extensive range of interferon stimulated genes (ISGs) with various anti-viral functions (reviewed here: [62]). In concurrence with previous studies of poxvirus-induced host responses [14, 15], Type I IFN responses were initiated by MVA, LSDV, CNPV and FWPV, with LSDV inducing the strongest response in mice (Fig. 4), followed by CNPV and FWPV, with MVA inducing a relatively low IFN response. FeP2 and PEPV induced very little ISG expression. The observed enhanced type I IFN-specific and other immune responses elicited by LSDV, FWPV and CNPV compared to MVA may be due to the absence of virus-encoded immunomodulators in these viruses which could still be present in MVA. Our results suggest that LSDV may be more immunogenic than FWPV and CNPV in mice. It is not known whether this greater IFN-response induced by LSDV in comparison to avipoxviruses would lead to enhanced clearance of the virus and a decreased immune response to any 


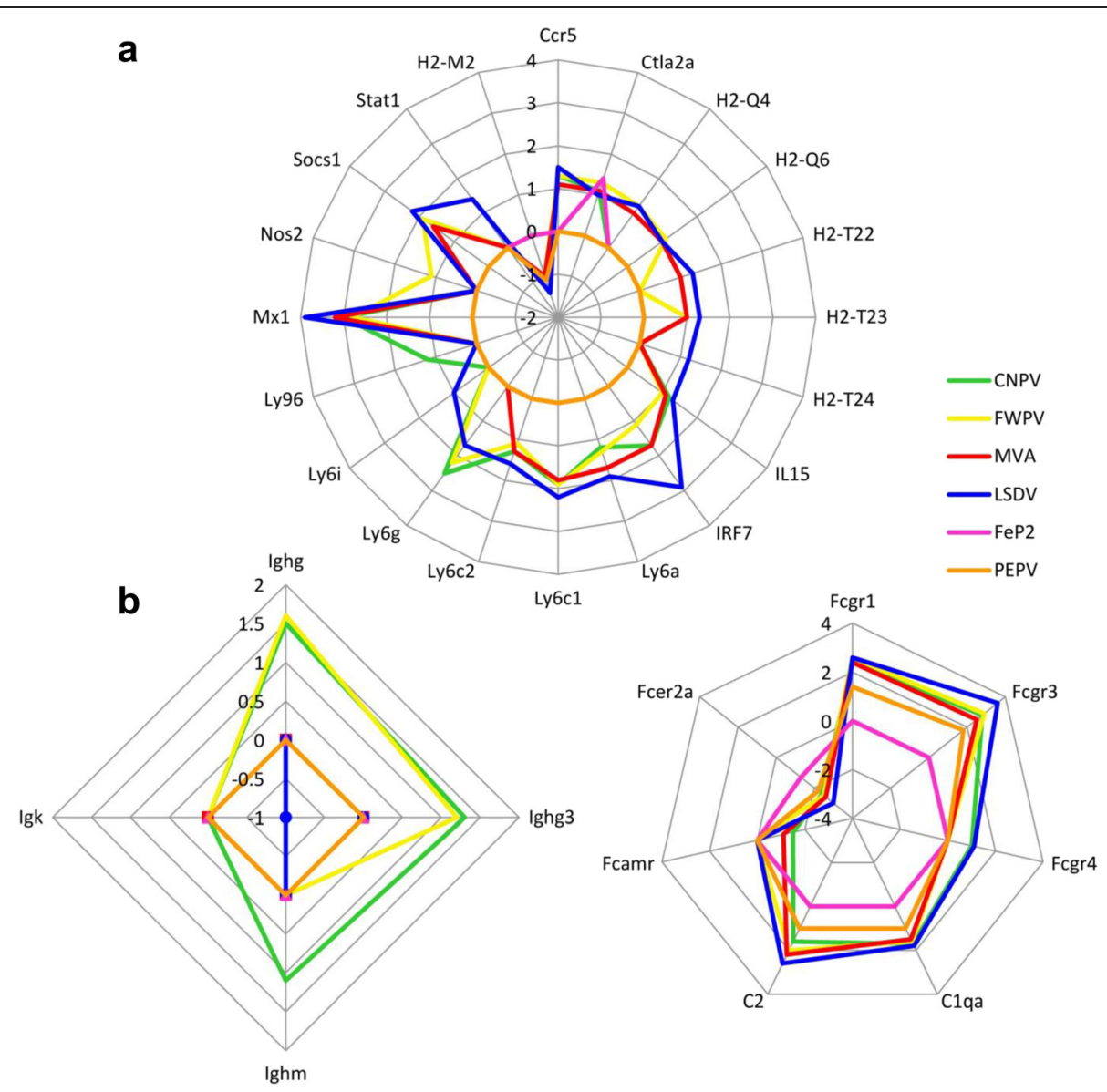

Fig. 4 a T-cell specific responses and 4 b) B-cell specific responses up- or down-regulated in mouse spleens in response to canarypox virus (CNPV), pigeonpox (FeP2), fowlpox virus (FWPV), lumpy skin disease virus (LSDV), modified vaccinia Ankara (MVA) and penguinpox virus (PEPV). The log 2 fold changes of significantly differentially expressed $(p$ value $\leq 0.05)$ genes involved in the respective types of responses are compared. A value of 0 indicates that no change was observed compared to mock infected mouse spleens. A positive value depicts upregulated genes and a negative value depicts down-regulated genes

potential transgenes, or whether the increased IFN response would result in an improved immune response to the transgene, should LSDV be used as a vaccine vector.

Toll-like receptors are important regulators of the innate immune system. Poxviruses are recognized by a number of different pathogen recognition receptors (PRRs) with innate immune sensing patterns differing considerably between species and even between different derivatives of the same parent species (VACV, MVA and NYVAC) [23]. In our study we show that TLR13, TLR3 and TLR8 are up-regulated by four poxviruses analysed (CNPV, FWPV, MVA and LSDV). In addition, TLR7 is up-regulated by CNPV, MVA and LSDV but not by FWPV (Table 2). Up regulation of TLR 3, which detects double stranded RNA, has been observed in response to MVA, but not NYVAC infection of MDDCs [14]. IFNs have been shown to up regulate TLR gene expression in viral infections [63]. Here we have established that CNPV, FWPV, MVA and LSDV all induce significant type I IFN responses and we suggest that the up regulation of TLR expression may be a result of this.

MVA, FWPV and CNPV all up regulate the protease Caspase 1 (Casp1) whereas LSDV does not (Fig. 4). Casp1 dependent programmed cell death (pyroptosis), unlike apoptosis, is a pro-inflammatory process that has recently been recognised as important for the control of microbial infections [64]. All of MVA, FWPV, CNPV and LSDV also significantly up-regulated caspase 4 (casp 4) (historically called caspase 11 in the mouse) (gene lists reference) which is required for the maturation of the pro-proteins of IL-1b and IL-18 (proIL-1b, proIL-18) and plays an important role in the activation of caspase- 1 in inflammasome complexes, and therefore inflammation [65]. The correlation of caspase up-regulation with either apoptosis or pyroptosis is still to be assessed.

Application of the systems biology approach to vaccines and determination of innate immune signatures has proven useful in predicting the immunogenicity of 
Table 4 Comparison of early poxvirus-induced immune responses to selected innate molecular signatures of existing vaccine vectors. Differences in $\log _{2}$ Fold Changes (between each virus and the control) are depicted

\begin{tabular}{|c|c|c|c|c|c|c|c|c|c|}
\hline & description & MVA & LSDV & CNPV & FWPV & PEPV & $\mathrm{FeP} 2$ & Evidence & References \\
\hline \multicolumn{10}{|c|}{ Innate immune response } \\
\hline \multicolumn{10}{|c|}{ Pathogen recognition } \\
\hline TIr13 & toll-like receptor 13 & 1.4 & 1.0 & 1.7 & 1.5 & 1.0 & - & & \\
\hline TIr3 & toll-like receptor 3 & 1 & 1.4 & 1.2 & 1.1 & - & - & Merck Ad5/HIV & {$[22]$} \\
\hline TIr7 & toll-like receptor 7 & 1.1 & 1.2 & 1.2 & - & - & - & YF-17D, LAIV & {$[20,21]$} \\
\hline Tlr8 & toll-like receptor 8 & 1.1 & 1.1 & 1.3 & 1.0 & - & - & Merck Ad5/HIV & {$[22]$} \\
\hline TIr11 & toll-like receptor 11 & -1.0 & - & - & - & - & - & & \\
\hline Ddx58 (RIG-I) & RIG-I-like receptor & - & 1.3 & - & - & - & - & YF-17D & {$[20]$} \\
\hline $\begin{array}{l}\text { Cd209a (DC } \\
\text { SIGN) }\end{array}$ & CD209a antigen & -1.7 & -2.2 & -1.1 & -1.5 & - & - & & \\
\hline $\begin{array}{l}\text { Cd209b (DC } \\
\text { SIGN) }\end{array}$ & CD209b antigen & -1.2 & -1.3 & - & - & - & - & & \\
\hline Ifih1 (MDA5) & RIG-I-like receptor & 1.3 & 2.1 & 1.2 & 1.2 & - & - & YF-17D & {$[20]$} \\
\hline Zbp1 (DAI) & $\begin{array}{l}\text { cytoplasmic double-stranded DNA } \\
\text { sensor }\end{array}$ & 1.9 & 2.7 & 1.3 & 1.6 & - & - & & \\
\hline Dhx58 (LGP2) & RIG-I-like receptor & 1.7 & 2.5 & 1.6 & 1.5 & - & - & YF-17D & {$[20]$} \\
\hline Eif2ak2 (PKR) & $\begin{array}{l}\text { eukaryotic translation initiation factor } \\
\text { 2-alpha kinase } 2 \text { (protein kinase R) }\end{array}$ & 1.1 & 2.1 & 1.1 & 1.1 & - & - & YF-17D & [20] \\
\hline \multicolumn{10}{|c|}{ Genes associated with the innate immune response of viral vectors } \\
\hline Cxcl10 (IP-10) & chemokine (C-X-C motif) ligand 10 & 2.7 & 3 & 2.2 & 2.8 & 1.7 & - & $\begin{array}{l}\text { Significantly upregulated in response } \\
\text { to YF-17D, Merck Ad5/HIV, TIV }\end{array}$ & [20-22] \\
\hline Mx1 & myxovirus (influenza virus) resistance 1 & 3.2 & 3.9 & 3 & 2.8 & - & - & YF-17D & [20] \\
\hline II-1a & interleukin 1 alpha & 1.9 & 2 & 2.2 & 2.3 & 1.8 & - & $\begin{array}{l}\text { Significantly upregulated in } \\
\text { response to YF-17D }\end{array}$ & \\
\hline Isg15 & ISG15 ubiquitin-like modifier & 1.6 & 2.2 & 1.5 & 1.4 & - & - & Merck Ad5/HIV & [22] \\
\hline Stat1 & $\begin{array}{l}\text { signal transducer and activator } \\
\text { of transcription } 1\end{array}$ & - & 1.4 & - & - & - & - & YF-17D, Merck Ad5/HIV, LAIV & [20-22] \\
\hline Cxcl11 (I-TAC) & chemokine (C-X-C motif) ligand 11 & 4.5 & 4.4 & 3.4 & 4.3 & 1.5 & - & Merck Ad5/HIV & {$[22]$} \\
\hline Ccr5 & chemokine (C-C motif) receptor 5 & 1.1 & 1.5 & 1.3 & 1.3 & - & - & Merck Ad5/HIV & [22] \\
\hline Gbp7 & guanylate binding protein 7 & 1.6 & 2.2 & 1.2 & 1.7 & - & - & Merck Ad5/HIV & [22] \\
\hline Irf1 & interferon regulatory factor 1 & - & 1.3 & - & 1.1 & - & - & Merck Ad5/HIV & [22] \\
\hline Stat2 & $\begin{array}{l}\text { signal transducer and activator } \\
\text { of transcription } 2\end{array}$ & 1.4 & 1.9 & 1.2 & 1.5 & - & - & LAIV & [21] \\
\hline Irf7 & interferon regulatory factor 7 & 1.7 & 2.9 & 1.7 & 1.1 & - & - & LAIV & [21] \\
\hline Casp1 & caspase 1 & 1.1 & - & 1.2 & 1.1 & - & - & & \\
\hline \multicolumn{10}{|c|}{ Adaptive immune response } \\
\hline \multicolumn{10}{|c|}{ B cell related responses } \\
\hline Ighg & $\begin{array}{l}\text { Immunoglobulin heavy chain } \\
\text { (gamma polypeptide) }\end{array}$ & - & - & 1.5 & 1.6 & - & - & & \\
\hline lghg3 & $\begin{array}{l}\text { Immunoglobulin heavy constant } \\
\text { gamma } 3\end{array}$ & - & - & 1.3 & 1.2 & - & - & $\begin{array}{l}\text { TIV, correlated with decreased risk of } \\
\text { HIV-1 infection in the RV144 trial } \\
\text { ALVAC-HIV(VCP1521) }\end{array}$ & \\
\hline Ighm & immunoglobulin heavy constant mu & - & - & 1.1 & - & - & - & $\begin{array}{l}\text { positively correllates with antibody } \\
\text { response to TIV }\end{array}$ & [21] \\
\hline Igk & $\begin{array}{l}\text { immunoglobulin kappa chain } \\
\text { complex }\end{array}$ & - & -1.0 & - & - & - & - & $\begin{array}{l}\text { positively correllates with antibody } \\
\text { response to TIV }\end{array}$ & [21] \\
\hline \multicolumn{10}{|c|}{$\mathrm{T}$ cell related responses } \\
\hline Gzmb & granzyme B & 3.7 & 4.7 & 4.1 & 4.2 & 2.4 & - & & [20] \\
\hline
\end{tabular}


Table 4 Comparison of early poxvirus-induced immune responses to selected innate molecular signatures of existing vaccine vectors. Differences in $\log _{2}$ Fold Changes (between each virus and the control) are depicted (Continued)

\begin{tabular}{|c|c|c|c|c|c|c|c|c|c|}
\hline & & & & & & & & $\begin{array}{l}\text { expressed by CD8+ T cells in } \\
\text { response to YF-17D }\end{array}$ & \\
\hline Ccr5 & chemokine ( $\mathrm{C}-\mathrm{C}$ motif) receptor 5 & 1.1 & 1.5 & 1.3 & 1.3 & - & - & $\begin{array}{l}\text { expressed by CD8+ T cells in } \\
\text { esponse to YF-17D }\end{array}$ & [20] \\
\hline Ccl2 (MCP1) & chemokine (C-C motif) ligand 2 & 3.5 & 3.3 & 2.9 & 3.3 & 2.8 & - & $\begin{array}{l}\text { predicted the magnitude of the CD8+ } \\
\text { T cell response to Merck Ad5/HIV }\end{array}$ & {$[22]$} \\
\hline
\end{tabular}

HIV human immunodeficiency virus, LAIV live attenuated influenza vaccine, TIV trivalent influenza vaccine, YF-17D Yellow fever vaccine, Merck Ad5/HIV Merck's Adenovirus subtype 5-based HIV vaccine

the highly effective yellow fever vaccine (YF-17D) [20], the seasonal influenza vaccines [21] and the immunogenic but inefficacious Merck Ad5/HIV vaccine [22]. Several of the innate immune signatures observed in tested vaccines, were common to one or more of the poxviruses investigated here. The gene encoding monocyte chemotactic protein 1 (MCP1) (Ccl2) was up-regulated by 5 out of the 6 poxviruses (MVA, LSDV,CNPV,FWPV and PEPV). This gene was positively correlated with the CD8+ T cell response to Merck Ad5/HIV vaccination [22]. Immunoglobulin genes, Ighm (up-regulated by CNPV) and IgK (down-regulated by LSDV) were positively correlated with the antibody response to TIV influenza vaccination [21]. This suggests that the different poxviruses could be associated with different levels of antibody induction during the adaptive immune response. Based on our data we speculate that LSDV may be more suitable for a T-cell based vaccine and CNPV more suitable for the induction of an antibody response. This reflects the published data on LSDV [3] and CNPV [61].

Microarray analyses can provide important information regarding the effect of different clinically relevant viruses on host gene expression. One limitation of microarray data analysis is that as of yet there are no standardised methods of statistical analysis. It has been demonstrated previously that fold change designations and $p$-value cutoffs can significantly alter microarray interpretation [66]. Here we have chosen stringent fold change and $p$-value cutoffs $\left(\log _{2} \mathrm{FC} \pm 1\right.$, adjusted $p$-value $\left.<0.05\right)$ in line with similar studies [11-14], in order to avoid false discovery and inaccurate biological inferences. We concede that in doing so, some smaller changes in gene expression may have been overlooked. Further work should entail investigating gene dysregulation at different times post infection. Also, innate immune signatures should be directly correlated with subsequent adaptive responses. Correlation of gene expression data with biological or clinical findings would be most informative.

\section{Conclusions}

The findings presented here indicate that six, genetically diverse host-restricted poxviruses, CNPV, FWPV, FeP2, PEPV, MVA and LSDV, produce qualitatively and quantitatively distinct host responses in an in vivo mouse model. These results confirm that transcriptome analysis in a mouse model can be used to determine if poxvirus vectors differ from each other, laying the ground work for further investigation.

\section{Materials and methods}

\section{Animal ethics approval}

The growth of poxviruses in embyonated eggs and the mouse experiments described below were approved by the Animal Research Ethics Committee in the Faculty of Health Sciences, University of Cape Town. The approval numbers are 013/016 and 013/017 respectively.

\section{Viruses}

MVA and wild-type CNPV were obtained from Prof. K. Dumbell's collection at the University of Cape Town and were originally from Prof. A. Mayr (Germany). The fowlpox virus vaccine, DCEP 25 modified strain, was purchased from Merial (Country) and LSDV vaccine, Herbivac ${ }^{\circ}$ (Ceva), was kindly donated by Deltamune (Pretoria, South Africa). FeP2 was from a Feral Pigeon (Columba livia) $[26,27]$ and PEPV from an African penguin (Spheniscus demersus) [27, 29]. Virus isolates were grown and titrated on the chorioallantoic membranes (CAMs) of embryonated 10-11 day old (MVA, CNPV, FWPV) or 7 day old (LSDV) Specific pathogen-free (SPF) White Leghorn chicken eggs, which were obtained from Avifarms (Pty) Ltd (Lyttelton, South Africa), using a method described previously [26]. Titrations were performed on CAMs for avipoxviruses and MVA and on Madin Darby bovine kidney (MDBK) cells for LSDV.

\section{Virus infection of mice}

Seven week old naive female BALB/c mice were randomly divided into groups of three and each mouse was inoculated intravenously (i.v) with $10^{5} \mathrm{pfu} / 100 \mathrm{ul}$ poxvirus, diluted in PBS or mock infected with PBS alone or egg extract $(100 \mu \mathrm{l})$. The egg extract was made from uninfected CAMs, following the same extraction and purification procedure as the virus samples above. We compared the gene expression profiles of the groups of mice that 
were mock-infected with egg extract and PBS. No difference in gene expression was observed between the control samples. For each different virus, three groups of three mice each were inoculated. At $24 \mathrm{~h}$ post infection, the mice were sacrificed by cervical dislocation without anaesthesia and the spleens were harvested and placed in RNAlater (Qiagen, Venlo, Limburg, NL).

\section{RNA extraction}

Mouse spleens were removed from RNAlater and the three spleens in each group were pooled and homogenized thoroughly using a TissueRuptor (Qiagen) in TRIzol ${ }^{\circ}$ reagent (Life Technologies, Carlsbad, CA, USA). Total RNA was isolated using TRIzol ${ }^{\circ}$ Plus RNA Purification Kit (Life Technologies, Carlsbad, CA, USA) with On-column PureLink DNase treatment according to manufacturer's instructions. RNA was resuspended in RNase free water and quality checked using the Nanodrop ND1000 (Thermoscientific, Waltham, MA, USA) and the Agilent Bioanalyzer Nano Assay (Agilent, Santa Clara, CA, USA).

\section{Microarray and data analysis}

mRNA hybridization was performed by IMGM Laboratories $\mathrm{GmbH}$ (Martinsried, DE) with the Affymetrix GeneChip Mouse Gene 2.0 ST array (Affymetrix, Santa Clara, CA, USA). Data analysis was performed in $\mathrm{R}$ [67], using packages from the Bioconductor suite (http://www.bioconductor.org), and CRAN (http://cran.rproject.org). All $\mathrm{R}$ code is available in Additional file 1. Probe level data from. CEL files was normalised using the Robust Multi-array Averaging (RMA) method [68] obtained as part of the "affy" package [69] from Bioconductor, resulting in $\log _{2}$ transformed values. Boxplots, scatterplots and histogram outputs of the normalised data were obtained and checked for consistency (not shown). Data was annotated using the Mouse Gene ST 2.0 annotation data package from Bioconductor. Nonspecific filtering was performed using the Genefilter package $[70,71]$. This step included an intensity filter which filtered the data set such that the intensity of each gene should be $>\log 2(100)$ in at least $20 \%$ of the samples. Secondly, a variance filter was applied such that the interquartile range of log2-intensities should be at least 0.5 .

Differential gene expression was determined using a linear model approach using the $\mathrm{R}$ package, Limma [34]. A heatmap was made using heatmap.2 from the CRAN package gplots [72], and depicted the unsupervised hierarchical clustering based on the genes with $p$-value $<0.05$ and $\log _{2} \mathrm{FC}$ above or below cutoff $( \pm 1)$. Venn diagrams were made using Venny [73] available at http://bioinfogp.cnb.csic.es/tools/venny/index.html. Functional analysis was performed using Database for Annotation, Visualization and Integrated Discovery
(DAVID) v6.7 web-based tools (http://david.abcc.ncifcrf.gov/tools.jsp). Quantitative real time PCR was done on selected mouse genes. GAPDH, HPRT and CD51 were selected as housekeeping genes; IRF7 and Zbp1 were selected as genes which were moderately upregulated by LSDV, MVA and avipoxviruses CNPV and FWPV; and IGFbp3 was selected as a gene which was downregulated by LSDV.

\section{Additional file}

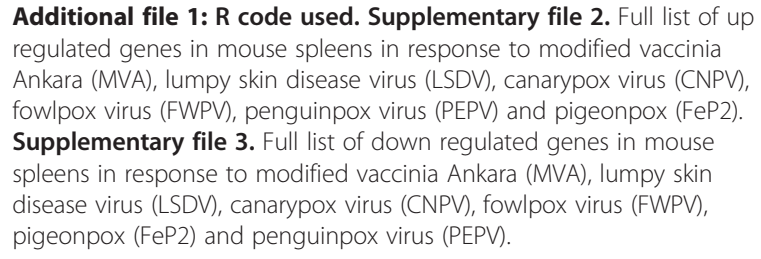

Additional file 1: $\mathbf{R}$ code used. Supplementary file 2 . Full list of up regulated genes in mouse spleens in response to modified vaccinia Ankara (MVA), lumpy skin disease virus (LSDV), canarypox virus (CNPV), fowlpox virus (FWPV), penguinpox virus (PEPV) and pigeonpox (FeP2). Supplementary file 3 . Full list of down regulated genes in mouse spleens in response to modified vaccinia Ankara (MVA), lumpy skin disease virus (LSDV), canarypox virus (CNPV), fowlpox virus (FWPV), pigeonpox (FeP2) and penguinpox virus (PEPV).

\section{Competing interests}

The authors declare that they have no competing interests.

\section{Authors' contributions}

ALW came up with the concept for the study. KO, OC, ND and ALW designed the study. KO did most of the experimental work. OC and ND provided the PEPV and LSDV seed stock respectively. RNA was isolated by $\mathrm{KO}, \mathrm{OC}$ and $\mathrm{ND}$. $\mathrm{KO}$ and $\mathrm{AD}$ performed the bioinformatics and data analysis $K O, A L W, A D, N D$ and RW participated in the interpretation of the data. KO wrote the initial manuscript. All authors read, edited and approved the final manuscript.

\section{Acknowledgments}

We thank Rodney Lucas of the UCT Research Animal Facility for technical assistance with the mouse experiments. This work is based on the research supported by the South African Research Chairs Initiative of the Department of Science and Technology and National Research Foundation, South Africa. Any opinion, finding and conclusion or recommendation expressed in this material is that of the authors and the NRF does not accept any liability in this regard. Financial support was also provided by the Carnegie Corporation of New York and Clinical Infectious Diseases Research Initiative (CIDRI) funded by the Wellcome Trust.

\section{Author details}

'Division of Medical Virology, Department of Clinical Laboratory Sciences, University of Cape Town, Cape Town, South Africa. ${ }^{2}$ Clinical Infectious Diseases Research Initiative, University of Cape Town, Cape Town, South Africa. ${ }^{3}$ Institute of Infectious Disease and Molecular Medicine, University of Cape Town, Cape Town, South Africa. ${ }^{4}$ Department of Medicine, University of Cape Town, Cape Town, South Africa. ${ }^{5}$ The Francis Crick Institute Mill Hill Laboratory, London NW7 1AA, UK. ${ }^{6}$ Department of Medicine, Imperial College, London W2 1PG, UK. ${ }^{7}$ National Health Laboratory Service, Groote Schuur Hospital, Cape Town, South Africa.

Received: 3 January 2015 Accepted: 28 May 2015

Published online: 08 July 2015

\section{References}

1. Garcia-Arriaza J, Esteban M. Enhancing poxvirus vectors vaccine immunogenicity. Hum Vaccin Immunother. 2014;10(8):2235-44.

2. Gomez CE, Perdiguero B, Garcia-Arriaza J, Esteban M. Poxvirus vectors as HIV/AIDS vaccines in humans. Hum Vaccin Immunother. 2012;8(9):1192-207.

3. Burgers WA, Ginbot Z, Shen YJ, Chege GK, Soares AP, Muller TL, et al. The novel capripoxvirus vector lumpy skin disease virus efficiently boosts modified vaccinia Ankara human immunodeficiency virus responses in rhesus macaques. J Gen Virol. 2014;95(Pt 10):2267-72. 
4. Draper SJ, Cottingham MG, Gilbert SC. Utilizing poxviral vectored vaccines for antibody induction-progress and prospects. Vaccine. 2013;31(39):4223-30.

5. Kim JW, Gulley JL. Poxviral vectors for cancer immunotherapy. Expert Opin Biol Ther. 2012;12(4):463-78.

6. Aspden K, Passmore JA, Tiedt F, Williamson AL. Evaluation of lumpy skin disease virus, a capripoxvirus, as a replication-deficient vaccine vector. J Gen Virol. 2003;84(Pt 8):1985-96.

7. Rerks-Ngarm S, Pitisuttithum P, Nitayaphan S, Kaewkungwal J, Chiu J, Paris $\mathrm{R}$, et al. Vaccination with ALVAC and AIDSVAX to prevent HIV-1 infection in Thailand. N Engl J Med. 2009;361(23):2209-20.

8. Baxby D, Paoletti E. Potential use of non-replicating vectors as recombinant vaccines. Vaccine. 1992;10(1):8-9.

9. Moss B. Vaccinia and other poxvirus expression vectors. Curr Opin Biotechnol. 1992;3(5):518-22.

10. Guerra S, Gonzalez JM, Climent N, Reyburn H, Lopez-Fernandez LA, Najera $J$, et al. Selective induction of host genes by MVA-B, a candidate vaccine against HIV/AIDS. J Virol. 2010;84(16):8141-52.

11. Guerra S, Lopez-Fernandez LA, Conde R, Pascual-Montano A, Harshman K, Esteban M. Microarray analysis reveals characteristic changes of host cell gene expression in response to attenuated modified vaccinia virus Ankara infection of human HeLa cells. J Virol. 2004;78(11):5820-34.

12. Guerra S, Lopez-Fernandez LA, Pascual-Montano A, Munoz M, Harshman K, Esteban M. Cellular gene expression survey of vaccinia virus infection of human HeLa cells. J Virol. 2003;77(11):6493-506.

13. Guerra S, Lopez-Fernandez LA, Pascual-Montano A, Najera JL, Zaballos A, Esteban M. Host response to the attenuated poxvirus vector NYVAC: upregulation of apoptotic genes and NF-kappaB-responsive genes in infected HeLa cells. J Virol. 2006;80(2):985-98.

14. Guerra S, Najera JL, Gonzalez JM, Lopez-Fernandez LA, Climent N, Gatell JM, et al. Distinct gene expression profiling after infection of immature human monocyte-derived dendritic cells by the attenuated poxvirus vectors MVA and NYVAC. J Virol. 2007:81(16):8707-21.

15. Harenberg A, Guillaume F, Ryan EJ, Burdin N, Spada F. Gene profiling analysis of ALVAC infected human monocyte derived dendritic cells. Vaccine. 2008;26(39):5004-13.

16. Teigler JE, Phogat S, Franchini G, Hirsch VM, Michael NL, Barouch DH. The canarypox virus vector ALVAC induces distinct cytokine responses compared to the vaccinia virus-based vectors MVA and NYVAC in rhesus monkeys. J Virol. 2014;88(3):1809-14.

17. Pacchioni S, Volonte L, Zanotto C, Pozzi E, De Giuli MC, Radaelli A. Canarypox and fowlpox viruses as recombinant vaccine vectors: an ultrastructural comparative analysis. Arch Virol. 2010;155(6):915-24.

18. Zanotto C, Pozzi E, Pacchioni S, Volonte L, De Giuli MC, Radaelli A. Canarypox and fowlpox viruses as recombinant vaccine vectors: a biological and immunological comparison. Antivir Res. 2010;88(1):53-63.

19. Takeuchi O, Akira S. Innate immunity to virus infection. Immunol Rev. 2009;227(1):75-86

20. Querec TD, Akondy RS, Lee EK, Cao W, Nakaya HI, Teuwen D, et al. Systems biology approach predicts immunogenicity of the yellow fever vaccine in humans. Nat Immunol. 2009;10(1):116-25.

21. Nakaya HI, Wrammert J, Lee EK, Racioppi L, Marie-Kunze S, Haining WN, et al. Systems biology of vaccination for seasonal influenza in humans. Nat Immunol. 2011;12(8):786-95.

22. Zak DE, Andersen-Nissen E, Peterson ER, Sato A, Hamilton MK, Borgerding J, et al. Merck Ad5/HIV induces broad innate immune activation that predicts CD8(+) T-cell responses but is attenuated by preexisting Ad5 immunity. Proc Natl Acad Sci U S A. 2012;109(50):E3503-12.

23. Lousberg EL, Diener KR, Brown MP, Hayball JD. Innate immune recognition of poxviral vaccine vectors. Expert Rev Vaccines. 2011;10(10):1435-49.

24. Johnson MJ, Petrovas C, Yamamoto T, Lindsay RW, Lore K, Gall JG, et al. Type I IFN induced by adenovirus serotypes 28 and 35 has multiple effects on T cell immunogenicity. J Immunol. 2012;188(12):6109-18.

25. Najera JL, Gomez CE, Domingo-Gil E, Gherardi MM, Esteban M. Cellular and biochemical differences between two attenuated poxvirus vaccine candidates (MVA and NYVAC) and role of the C7L gene. J Virol. 2006;80(12):6033-47.

26. Offerman K, Carulei O, Gous TA, Douglass N, Williamson AL. Phylogenetic and histological variation in avipoxviruses isolated in South Africa. J Gen Virol. 2013;94(Pt 10):2338-51.

27. Offerman K, Carulei O, van der Walt AP, Douglass N, Williamson AL. The complete genome sequences of poxviruses isolated from a penguin and a pigeon in South Africa and comparison to other sequenced avipoxviruses. BMC Genomics. 2014;15:463.

28. Stannard LM, Marais D, Kow D, Dumbell KR. Evidence for incomplete replication of a penguin poxvirus in cells of mammalian origin. J Gen Virol. 1998;79(Pt 7):1637-46.

29. Carulei O, Douglass N, Williamson AL. Phylogenetic analysis of three genes of Penguinpox virus corresponding to Vaccinia virus G8R (VLTF-1), A3L (P4b) and $\mathrm{H} 3 \mathrm{~L}$ reveals that it is most closely related to Turkeypox virus, Ostrichpox virus and Pigeonpox virus. Virol J. 2009;6:52.

30. Rubins KH, Hensley LE, Relman DA, Brown PO. Stunned silence: gene expression programs in human cells infected with monkeypox or vaccinia virus. PLoS One. 2011;6(1):e15615.

31. Brum LM, Lopez MC, Varela JC, Baker HV, Moyer RW. Microarray analysis of A549 cells infected with rabbitpox virus (RPV): a comparison of wild-type RPV and RPV deleted for the host range gene, SPI-1. Virology. 2003;315(2):322-34.

32. Matsumiya T, Stafforini DM. Function and regulation of retinoic acid-inducible gene-I. Crit Rev Immunol. 2010;30(6):489-513.

33. Ruffell D, Mourkioti F, Gambardella A, Kirstetter P, Lopez RG, Rosenthal N, et al. A CREB-C/EBPbeta cascade induces M2 macrophage-specific gene expression and promotes muscle injury repair. Proc Natl Acad Sci U S A. 2009;106(41):17475-80.

34. Poli $\mathrm{V}$. The role of $\mathrm{C} / \mathrm{EBP}$ isoforms in the control of inflammatory and native immunity functions. J Biol Chem. 1998;273(45):29279-82.

35. Abele R, Tampe R. Function of the transport complex TAP in cellular immune recognition. Biochim Biophys Acta. 1999;1461(2):405-19.

36. Petit V, Guetard D, Renard M, Keriel A, Sitbon M, Wain-Hobson S, et al. Murine APOBEC1 is a powerful mutator of retroviral and cellular RNA in vitro and in vivo. J Mol Biol. 2009;385(1):65-78.

37. Ghosh S, Gregory D, Smith A, Kobzik L. MARCO regulates early inflammatory responses against influenza: a useful macrophage function with adverse outcome. Am J Respir Cell Mol Biol. 2011;45(5):1036-44.

38. Coelho AL, Schaller MA, Benjamim CF, Orlofsky AZ, Hogaboam CM, Kunkel SL. The chemokine CCL6 promotes innate immunity via immune cell activation and recruitment. J Immunol. 2007;179(8):5474-82.

39. Kim YG, Park JH, Reimer T, Baker DP, Kawai T, Kumar H, et al. Viral infection augments Nod1/2 signaling to potentiate lethality associated with secondary bacterial infections. Cell Host Microbe. 2011;9(6):496-507.

40. Chen SC, Vassileva G, Kinsley D, Holzmann S, Manfra D, Wiekowski MT, et al. Ectopic expression of the murine chemokines CCL21a and CCL21b induces the formation of lymph node-like structures in pancreas, but not skin, of transgenic mice. J Immunol. 2002;168(3):1001-8.

41. Shimizu Y, Honda S, Yotsumoto K, Tahara-Hanaoka S, Eyre HJ, Sutherland $\mathrm{GR}$, et al. Fc(alpha)/mu receptor is a single gene-family member closely related to polymeric immunoglobulin receptor encoded on Chromosome 1. Immunogenetics. 2001;53(8):709-11.

42. Baalasubramanian S, Harris CL, Donev RM, Mizuno M, Omidvar N, Song WC, et al. CD59a is the primary regulator of membrane attack complex assembly in the mouse. J Immunol. 2004;173(6):3684-92.

43. Sempowski GD, Lee DM, Kaufman RE, Haynes BF. Structure and function of the CD7 molecule. Crit Rev Immunol. 1999;19(4):331-48.

44. Rusinova I, Forster S, Yu S, Kannan A, Masse M, Cumming H, et al. Interferome v2.0: an updated database of annotated interferon-regulated genes. Nucleic Acids Res. 2013;41(Database issue):D1040-6.

45. Der SD, Zhou A, Williams BR, Silverman RH. Identification of genes differentially regulated by interferon alpha, beta, or gamma using oligonucleotide arrays. Proc Natl Acad Sci U S A. 1998;95(26):15623-8.

46. Schlaak JF, Hilkens CM, Costa-Pereira AP, Strobl B, Aberger F, Frischauf AM, et al. Cell-type and donor-specific transcriptional responses to interferon-alpha. Use of customized gene arrays. J Biol Chem. 2002;277(51):49428-37.

47. Querec T, Bennouna S, Alkan S, Laouar Y, Gorden K, Flavell R, et al. Yellow fever vaccine YF-17D activates multiple dendritic cell subsets via TLR2, 7, 8, and 9 to stimulate polyvalent immunity. J Exp Med. 2006;203(2):413-24.

48. Pulendran B. Variegation of the immune response with dendritic cells and pathogen recognition receptors. J Immunol. 2005;174(5):2457-65.

49. Somogyi P, Frazier J, Skinner MA. Fowlpox virus host range restriction: gene expression, DNA replication, and morphogenesis in nonpermissive mammalian cells. Virology. 1993;197(1):439-44.

50. Offerman K. Investigation of local South African avipoxviruses as potential vaccine vectors. Cape Town: University of Cape Town; 2014 
51. Taylor J, Meignier B, Tartaglia J, Languet B, VanderHoeven J, Franchini G, et al. Biological and immunogenic properties of a canarypox-rabies recombinant, ALVAC-RG (VCP65) in non-avian species. Vaccine. 1995;13(6):539-49.

52. Tulman ER, Afonso CL, Lu Z, Zsak L, Kutish GF, Rock DL. The genome of canarypox virus. J Virol. 2004;78(1):353-66.

53. Bourquain D, Dabrowski PW, Nitsche A. Comparison of host cell gene expression in cowpox, monkeypox or vaccinia virus-infected cells reveals virus-specific regulation of immune response genes. Virol J. 2013;10:61

54. Bahar MW, Kenyon JC, Putz MM, Abrescia NG, Pease JE, Wise EL, et al. Structure and function of A41, a vaccinia virus chemokine binding protein. PLoS Pathog. 2008:4(1):e5.

55. Clark RH, Kenyon JC, Bartlett NW, Tscharke DC, Smith GL. Deletion of gene A41L enhances vaccinia virus immunogenicity and vaccine efficacy. J Gen Virol. 2006;87(Pt 1):29-38

56. Shen YJ, Shephard E, Douglass N, Johnston N, Adams C, Williamson C, et al. A novel candidate HIV vaccine vector based on the replication deficient Capripoxvirus, Lumpy skin disease virus (LSDV). Virol J. 2011;8:265.

57. Lue H, Kleemann R, Calandra T, Roger T, Bernhagen J. Macrophage migration inhibitory factor (MIF): mechanisms of action and role in disease. Microbes Infect/Institut Pasteur. 2002;4(4):449-60.

58. Takeuchi O, Akira S. MDA5/RIG-I and virus recognition. Curr Opin Immunol. 2008;20(1):17-22.

59. Luscher B. Function and regulation of the transcription factors of the Myc/ Max/Mad network. Gene. 2001;277(1-2):1-14.

60. Wang X, Han Y, Dang Y, Fu W, Zhou T, Ptak RG, et al. Moloney leukemia virus 10 (MOV10) protein inhibits retrovirus replication. J Biol Chem. 2010;285(19):14346-55.

61. Yates NL, Liao HX, Fong Y, de Camp A, Vandergrift NA, Williams WT, et al. Vaccine-induced Env V1-V2 lgG3 correlates with lower HIV-1 infection risk and declines soon after vaccination. Sci Transl Med. 2014;6(228):228ra239.

62. Liu SY, Sanchez DJ, Cheng G. New developments in the induction and antiviral effectors of type I interferon. Curr Opin Immunol. 2011;23(1):57-64.

63. Miettinen M, Sareneva T, Julkunen I, Matikainen S. IFNs activate toll-like receptor gene expression in viral infections. Genes Immun. 2001;2(6):349-55.

64. Bergsbaken T, Fink SL, Cookson BT. Pyroptosis: host cell death and inflammation. Nat Rev Microbiol. 2009;7(2):99-109.

65. Sollberger G, Strittmatter GE, Kistowska M, French LE, Beer HD. Caspase-4 is required for activation of inflammasomes. J Immunol. 2012;188(4):1992-2000.

66. Dalman MR, Deeter A, Nimishakavi G, Duan ZH. Fold change and p-value cutoffs significantly alter microarray interpretations. BMC Bioinformatics. 2012;13 Suppl 2:S11

67. Team RC. R: a language and environment for statistical computing. Vienna: R Foundation for Statistical Computing; 2012. 2013. Available from: http:// www.R-project.org. ISBN 3-900051-07-0.

68. Irizarry RA, Hobbs B, Collin F, Beazer-Barclay YD, Antonellis K, Scherf U, et al. Exploration, normalization, and summaries of high density oligonucleotide array probe level data. Biostatistics. 2003;4(2):249-64.

69. Gautier L, Cope L, Bolstad BM, Irizarry RA. affy-analysis of Affymetrix GeneChip data at the probe level. Bioinformatics. 2004;20(3):307-15.

70. Gentleman R, Carey V, Huber W, Hahne F. Genefilter: Methods for filtering genes from microarray experiments. R package version 2011, 1(0). http:// www.bioconductor.org/packages/release/bioc/html/genefilter.html.

71. Smyth GK. Limma: linear models for microarray data. In: Bioinformatics and computational biology solutions using R and Bioconductor. New York: Springer; 2005. p. 397-420.

72. Warnes GR, Bolker B, Bonebakker L, Gentleman R, Huber W, Liaw A, Lumley T, Maechler M, Magnusson A, Moeller S. gplots: Various R programming tools for plotting data. R package version 2009, 2(4). http://cran.r-project.org/web/packages/gplots/gplots.pdf.

73. Oliveros JC. VENNY. An interactive tool for comparing lists with Venn Diagrams. 2007.

\section{Submit your next manuscript to BioMed Central and take full advantage of:}

- Convenient online submission

- Thorough peer review

- No space constraints or color figure charges

- Immediate publication on acceptance

- Inclusion in PubMed, CAS, Scopus and Google Scholar

- Research which is freely available for redistribution 